\title{
ON THE GEOMETRIC ANDRÉ-OORT CONJECTURE FOR VARIATIONS OF HODGE STRUCTURES
}

\author{
JIAMING CHEN
}

\begin{abstract}
Авsтract. Let $\mathbb{V}$ be a polarized variation of integral Hodge structure on a smooth complex quasi-projective variety $S$. In this paper, we show that the union of the non-factor special subvarieties for $(S, \mathbb{V})$, which are of Shimura type with dominant period maps, is a finite union of special subvarieties of $S$. This generalizes previous results of Clozel and Ullmo [CU05-1], Ullmo [Ull07] on the distribution of the non-factor (in particular, strongly) special subvarieties in a Shimura variety to the non-classical setting and also answers positively the geometric part of a conjecture of Klingler on the André-Oort conjecture for variations of Hodge structures.
\end{abstract}

\section{INTRODUCTION}

1.1. Motivation. The classical André-Oort conjecture, which describes the distribution of CM points (points with complex multiplication) on a Shimura variety, asserts that the Zariski closure of a subset of CM points in a Shimura variety is special (namely, an irreducible component of a Hecke translate of a Shimura subvariety). It is the analog in a Hodge-theoretic context of the Manin-Mumford conjecture (a theorem of Raynaud ([Ray88]) stating that an irreducible subvariety of a complex abelian variety containing a Zariski-dense set of torsion points is the torsion translate of an abelian subvariety. It has recently been proved for the Shimura variety $\mathcal{A}_{g}$ moduli space of principally polarized complex abelian varieties of dimension $g$ (and more generally for mixed Shimura varieties whose pure part are of abelian type) following a strategy proposed by Pila and Zannier, through the work of many authors ([PT14], [KUY16], [Gao17], [AGHM18], [YZ18], [Tsi18]). Recently, Klingler ([K17]) formulated a generalization of the André-Oort conjecture (in fact, of the more general ZilberPink's conjecture on atypical intersections in Shimura varieties) for any admissible variation of mixed Hodge structures on a smooth quasi-projective variety.

This paper studies a particular case of Klingler's generalized André-Oort conjecture for pure variations of integral Hodge structures.

1.2. Hodge locus. Let $\mathbb{V} \rightarrow S^{\text {an }}$ be a polarized ${ }^{1}$ variation of integral Hodge structure (usually abbreviated $\mathbb{Z}$-VHS) of weight $p \in \mathbb{Z}$ on a smooth irreducible complex quasi-projective variety $S$. Thus $\mathbb{V}$ is a triple $\left(\mathbb{V}_{\mathbb{Z}}, F^{\bullet}, \varphi\right)$, where $\mathbb{V}_{\mathbb{Z}}$ is a local system of finite free $\mathbb{Z}$-modules

\footnotetext{
${ }^{1}$ We only consider polarizable variations of Hodge structures throughout the paper.
} 
on the complex manifold $S^{\text {an }}$ - the analytification of $S, F^{\bullet}$ is a decreasing filtration by holomorphic subbundles on the holomorphic bundle $\left(\mathcal{V}^{\text {an }}:=\mathbb{V}_{\mathbb{Z}} \otimes_{\underline{\mathbb{Z}}_{\text {sa }}} \mathcal{O}_{S^{\mathrm{an}}}, \nabla^{\text {an }}\right)$ satisfying Griffiths' transversality condition

$$
\nabla^{\text {an }} F^{\bullet} \subset \Omega_{S^{\text {an }}}^{1} \otimes_{\mathcal{O}_{S \text { an }}} F^{\bullet-1}
$$

and $\varphi: \mathbb{V}_{\mathbb{Z}} \otimes \mathbb{V}_{\mathbb{Z}} \rightarrow \underline{\mathbb{Z}}_{S \text { an }}(-p)$ is a bilinear pairing of local systems, such that $\left(\mathbb{V}_{\mathbb{Z}, s}, F_{s}^{\bullet}, \varphi_{s}\right)$ is a polarized $\mathbb{Z}$-Hodge structure of weight $p$ for all $s \in S^{\text {an }}$. This definition is an abstraction of the geometric case corresponding to $\mathbb{V}_{\mathbb{Z}}=\left(R^{p} f_{*} \mathbb{Z}_{\mathcal{X}} \text { an }\right)_{\text {prim }} /$ torsion (for $p \geq 0$ ), the primitive part of the local system of the $p$-th integral cohomologies modulo torsion of the fibers of a smooth projective morphism $f: \mathcal{X} \rightarrow S$ and $\left(\mathcal{V}^{\text {an }}, \nabla^{\text {an }}\right)$ the Gauss-Manin connection. Following Griffiths [cf. [Sch73] Theorem (4.13)] the holomorphic bundle $\mathcal{V}^{\text {an }}$ admits a unique algebraic structure $\mathcal{V}$ such that the holomorphic connection $\nabla^{\text {an }}$ is the analytification of an algebraic connection $\nabla$ on $\mathcal{V}$ which is regular, and the filtration $F^{\bullet} \mathcal{V}^{\text {an }}$ is the analytification of an algebraic filtration $F^{\bullet} \mathcal{V}$. Thus from now on we will omit an from the notations and the meaning will be clear from the context.

Inspired by the rational Hodge conjecture, one would like to know how the Hodge locus $\operatorname{HL}\left(S, \mathbb{V}^{\otimes}\right) \subset S$ is distributed in $S$. Here $\operatorname{HL}\left(S, \mathbb{V}^{\otimes}\right)$ is by definition the subset of points $s$ of $S$ for which exceptional Hodge classes ${ }^{2}$ do occur in $\mathbb{V}_{\mathbb{Q}, s}^{m} \otimes\left(\mathbb{V}_{\mathbb{Q}, s}^{\vee}\right)^{n}$ for some $m, n \in \mathbb{Z}_{>0}$, where $\mathbb{V}_{\mathbb{Q}, s}^{\vee}$ denotes the $\mathbb{Q}$-Hodge structure dual to $\mathbb{V}_{\mathbb{Q}, s}$.

The Tannakian formalism available for Hodge structures gives us a particularly useful group-theoretic description of the Hodge locus $\operatorname{HL}(S, \mathbb{V} \otimes)$. Recall that for every $s \in S$, the Mumford-Tate group $G_{s}$ of the Hodge structure $\mathbb{V}_{\mathbb{Q}, s}$ is the Tannakian group of the Tannakian subcategory $<\mathbb{V}_{\mathbb{Q}, s}^{\otimes}>$ of pure polarized Hodge structures tensorially generated by $\mathbb{V}_{\mathbb{Q}, s}$ and $\mathbb{V}_{\mathbb{Q}, s}^{\vee}$. Equivalently, the group $\boldsymbol{G}_{s}$ is the stabilizer of the Hodge classes in the rational Hodge structures tensorially generated by $\mathbb{V}_{\mathbb{Q}, s}$ and its dual. The group $G_{s}$ is a connected reductive algebraic $\mathbb{Q}$-group, canonically endowed with a morphism of real algebraic groups $h_{s}: \mathbb{S}:=\operatorname{Res}_{\mathbb{C} / \mathbb{R}} \mathbb{G}_{m} \rightarrow \boldsymbol{G}_{s, \mathbb{R}}$. Let $Z \subset S$ be an irreducible algebraic subvariety of $S$. A point $s$ in the smooth locus $Z^{\text {sm }}$ of $Z$ is said to be Hodge generic for the restriction $\left.\mathbb{V}\right|_{Z^{\text {sm }}}$ if $G_{s}$ is maximal when $s$ ranges through $Z^{\text {sm }}$. Since $Z$ is irreducible, two Hodge generic points of $Z^{\text {sm }}$ have the same Mumford-Tate group, called the generic Mumford-Tate group $G_{Z}$ of $\left(Z,\left.\mathbb{V}\right|_{Z^{\text {sm }}}\right)$. Then the Hodge locus $\operatorname{HL}\left(S, \mathbb{V}^{\otimes}\right)$ is also the subset of points of $S$ which are not Hodge generic.

A fundamental result of Cattani-Deligne-Kaplan [CDK95] states that $\operatorname{HL}\left(S, \mathbb{V}^{\otimes}\right)$ is a countable union of closed irreducible strict algebraic subvarieties of $S$.

\footnotetext{
${ }^{2}$ In this paper, by a Hodge class, we will always mean a Hodge class of type $(0,0)$.
} 
Definition 1.1. Let $\mathbb{V}$ be a $\mathbb{Z}$-VHS on a smooth irreducible complex quasi-projective variety $S$.

A closed irreducible algebraic subvariety $Z$ of $S$ is called special for $\mathbb{V}$, if it is maximal among the closed irreducible algebraic subvarieties of $S$ with the same generic MumfordTate group as $Z$.

Special subvarieties of dimension zero are called special points for $(S, \mathbb{V})$.

A special point $s \in S$ whose Mumford-Tate group $G_{s}$ is commutative is called a CM point for $(S, \mathbb{V})$.

So by definition, if $Z \subset S$ is a special subvariety for $(S, \mathbb{V})$, then $Z$ is either contained in $\operatorname{HL}\left(S, \mathbb{V}^{\otimes}\right)$ (in which case we call $Z$ strict), or $Z=S$.

Choose $s \in Z^{\text {sm }}$ and let $\mathcal{D}_{Z}$ be the $G_{Z}(\mathbb{R})$-conjugacy class of $h_{s}$. The pair $\left(\boldsymbol{G}_{Z}, \mathcal{D}_{Z}\right)$ is called the generic Hodge datum for $\left(Z,\left.\mathbb{V}\right|_{Z^{\text {sm }}}\right)$.

Definition 1.2. Let $Z \subset S$ be a special subvariety for $\mathbb{V}$. Then $Z$ is called of Shimura type if the generic Hodge datum $\left(\boldsymbol{G}_{Z}, \mathcal{D}_{Z}\right)$ for $\left(Z,\left.\mathbb{V}\right|_{Z^{\text {sm }}}\right)$ is a Shimura datum (see [Mil05] Section 5 for the definition of Shimura datum).

Notice that $\mathrm{CM}$ points for $(S, \mathbb{V})$ are of Shimura type.

The problem we are interested in can be phrased vaguely as follows:

Question 1.3. Given a $\mathbb{Z}-\mathrm{VHS}$ on a smooth irreducible complex quasi-projective variety, can we describe the distribution of its CM points, or more generally of its special subvarieties of Shimura type?

\subsection{André-Oort conjecture for variations of Hodge structures.}

1.3.1. Variations of Hodge structures of Shimura type and of general Hodge type. We keep the same notations as in the previous section. The Griffiths' transversality condition (1) establishes a fundamental dichotomy between $\mathbb{Z}$-VHS of Shimura type (called classical in [GGK12]) for which the generic Hodge datum $(\boldsymbol{G}, \mathcal{D})$ is a Shimura datum and $\mathbb{Z}$-VHS of general Hodge type (called non-classical in [GGK12]). Roughly speaking, a $\mathbb{Z}-\mathrm{VHS}$ is of Shimura type if it is an element of some $\left\langle\mathbb{V}_{\mathbb{Q}}^{\otimes}>\right.$, for $\mathbb{V}$ an effective $\mathbb{Z}$-VHS of weight $p=1$ (i.e., a family of abelian varieties), or weight $p=2$ and very restricted Hodge type (like family of K3surfaces). It is the Hodge-theoretic incarnation of a family of abelian motives. On the other hand, variations of integral Hodge structures of general Hodge type form the vast majority of $\mathbb{Z}$-VHS, incarnating families of non-abelian motives.

For a $\mathbb{Z}$-VHS of Shimura type, the Hodge filtration is so short that the Griffiths' transversality condition is automatically satisfied. As a result, classifying spaces do exist for $\mathbb{Z}$-VHS 
of Shimura type: these are exactly the Shimura varieties $\operatorname{Sh}_{K}(\boldsymbol{G}, \mathcal{D})$ (see [Mil05] Section 5 for the definition of a Shimura datum), which are algebraic varieties (canonically defined over a number field) generalizing the moduli space $\mathcal{A}_{g}$ of principally polarized abelian varieties of dimension $g$. Given $\mathbb{V}$ a $\mathbb{Z}$-VHS of Shimura type on $S$, there exists an algebraic classifying map $\psi: S \rightarrow \operatorname{Sh}_{K}(\boldsymbol{G}, \mathcal{D})$ and an algebraic representation $\rho$ of $\boldsymbol{G}$ such that $\mathbb{V}=\psi^{*} \mathbb{V}_{\rho}$. Here $\mathbb{V}_{\rho}$ is the standard $\mathbb{Z}$-VHS on $\operatorname{Sh}_{K}(\boldsymbol{G}, \mathcal{D})$ associated to $\rho$. Moreover the Hodge locus $\operatorname{HL}\left(S, \mathbb{V}^{\otimes}\right)$ coincides with $\psi^{-1}\left(\psi(S) \cap \operatorname{HL}\left(\operatorname{Sh}_{K}(\boldsymbol{G}, \mathcal{D})\right)\right.$, where the Hodge locus $\operatorname{HL}\left(\operatorname{Sh}_{K}(\boldsymbol{G}, \mathcal{D})\right):=\operatorname{HL}\left(\operatorname{Sh}_{K}(\boldsymbol{G}, \mathcal{D}), \mathbb{V}_{\rho}^{\otimes}\right)$ is in fact independent of the choice of the faithful representation $\rho$ of $\boldsymbol{G}$ and each special subvariety of $\mathrm{Sh}_{K}(\boldsymbol{G}, \mathcal{D})$ can be geometrically described as an irreducible component of a Hecke translate of a Shimura subvariety of $\mathrm{Sh}_{K}(\boldsymbol{G}, \mathcal{D})$.

1.3.2. The conjectures. The following conjecture of Klingler proposes a characterization of the $\mathbb{Z}$-VHS with many CM points.

Conjecture 1.4 (Klingler [K17], Conjecture 5.3). Let $\mathbb{V}$ be a $\mathbb{Z}-V H S$ on a smooth irreducible complex quasi-projective variety $S$ with generic Hodge datum $(G, \mathcal{D})$. Suppose that the set of $C M$ points for $(S, \mathbb{V})$ is Zariski-dense in $S$. Then $(\boldsymbol{G}, \mathcal{D})$ is a Shimura datum and we have a Cartesian diagram

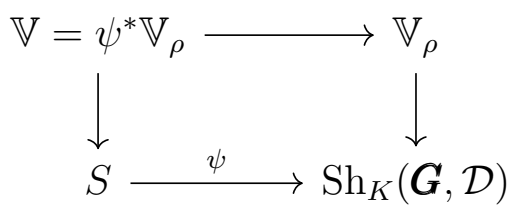

where $\psi$ is a dominant morphism to a connected component $\operatorname{Sh}_{K}^{\circ}(\boldsymbol{G}, \mathcal{D})$ of a Shimura variety $\operatorname{Sh}_{K}(\boldsymbol{G}, \mathcal{D})$, $\rho: G \rightarrow \mathbf{G L}(V)$ is an algebraic representation and $\mathbb{V}_{\rho} \rightarrow \operatorname{Sh}_{K}(\boldsymbol{G}, \mathcal{D})$ is the associated standard $\mathbb{Z}$ VHS on $\operatorname{Sh}_{K}(\boldsymbol{G}, \mathcal{D})$.

It follows readily from these considerations that the restriction of conjecture 1.4 to the class of $\mathbb{Z}$-VHS of Shimura type is equivalent to the classical André-Oort conjecture, while the full conjecture 1.4 is equivalent to both the classical André-Oort Conjecture and the following conjecture 1.5:

Conjecture 1.5. Let $\mathbb{V}$ be a $\mathbb{Z}-V H S$ on a smooth irreducible complex quasi-projective variety $S$. Suppose that the set of $C M$ points for $(S, \mathbb{V})$ is Zariski-dense in $S$. Then $(S, \mathbb{V})$ is of Shimura type.

Many works have been devoted to the classical André-Oort conjecture, culminating to its proof when a Shimura variety $\operatorname{Sh}_{K}(\boldsymbol{G}, X)$ is of abelian type (see for example [KUY18] for a survey). The proof of the classical André-Oort Conjecture relies on two completely different ingredients: on the one hand a precise arithmetic analysis of the Galois orbits of CM points (lower bound and heights); on the other hand, a geometric analysis of the distribution in $\mathrm{Sh}_{K}(\boldsymbol{G}, X)$ of positive dimensional special subvarieties. 
In this paper we will concentrate on the geometric part of conjecture 1.5, namely on the following:

Conjecture 1.6 (geometric André-Oort for $\mathbb{Z}-\mathrm{VHS}$, Klingler [K17], Conjecture 5.7). Let $\mathbb{V}$ be a $\mathbb{Z}-V H S$ on a smooth irreducible complex quasi-projective variety $S$. Suppose that the set of positive dimensional special subvarieties for $(S, \mathbb{V})$, which are of Shimura type with dominant period maps, is Zariski-dense in $S$. Then $(S, \mathbb{V})$ is of Shimura type with dominant period map.

1.4. Statements of the main results. The main result we obtain in the direction of Conjecture 1.6 is the following:

Theorem 1.7. Let $\mathbb{V}$ be a $\mathbb{Z}-V H S$ on a smooth irreducible complex quasi-projective variety $S$. Then the union of the non-factor special subvarieties for $(S, \mathbb{V})$, which are of Shimura type with dominant period maps, is a finite union of special subvarieties of $S$.

As a corollary, we have

Corollary 1.8. Let $\mathbb{V}$ be a $\mathbb{Z}-V H S$ on a smooth irreducible complex quasi-projective variety $S$. If $S$ contains a Zariski-dense subset of non-factor special subvarieties, which are of Shimura type with dominant period maps, then $(S, \mathbb{V})$ is of Shimura type with dominant period map.

Remark 1.9. The notion of non-factor special subvarieties was introduced by Ullmo in [Ull07] for Shimura varieties, as a generalization of the strongly special subvarieties defined by Clozel and Ullmo in [CU05-1]. The precise definition is given in Section 4. The restriction to non-factor special subvarieties avoids in particular the appearance of the special points. We have no tools to deal with special points in the non-classical setting at the moment.

Remark 1.10. Recent work of Klingler and Otwinowska [KO19] shows that if the adjoint group $G^{\text {ad }}$ of the generic Mumford-Tate group $\boldsymbol{G}$ of $(S, \mathbb{V})$ is simple, then the union of positive special subvarieties in $\mathrm{HL}\left(S, \mathbb{V}^{\otimes}\right)$ is either an algebraic subvariety of $S$ or is Zariskidense in $S$. Here we say an irreducible algebraic subvariety $\mathrm{Z}$ of $S$ is positive if the local system $\left.\mathbb{V}\right|_{Z}$ is not constant.

Theorem 1.7 is a consequence of the following equidistribution result of non-factor Shimura type special subvarieties in any connected Hodge variety, which is a generalization to the non-classical setting of Clozel and Ullmo's [CU05-1] and Ullmo's [Ull07] result on the equidistribution of positive dimensional special subvarieties in a Shimura variety.

Theorem 1.11. Let $\mathbb{V}$ be a $\mathbb{Z}-V H S$ on a smooth irreducible complex quasi-projective variety $S$ with generic Hodge datum $(\boldsymbol{G}, \mathcal{D})$. Let $\psi: S \rightarrow \operatorname{Hod}_{\Gamma}(S, \mathbb{V}):=\Gamma \backslash \mathcal{D}$ be the associated period map, where $\Gamma \subset G(\mathbb{Q})$ is an arithmetic lattice.

Let $\left(Z_{n}\right)$ be a sequence of non-factor special subvariety of $S$ which are of Shimura type with dominant period maps and $\left(W_{n}\right)$ be the corresponding sequence of non-factor Shimura type special subvarieties in $\operatorname{Hod}_{\Gamma}(S, \mathbb{V})$. Let $\mu_{W_{n}}$ be the canonical Borel probability measure on $\operatorname{Hod}_{\Gamma}(S, \mathbb{V})$ 
with support $W_{n}$. Then there exists a special subvariety $W_{\infty}$ of $\operatorname{Hod}_{\Gamma}(S, \mathbb{V})$, which is non-factor and of Shimura type, and a subsequence $\left(\mu_{W_{n_{k}}}\right)$ of $\left(\mu_{W_{n}}\right)$ such that $\mu_{W_{n_{k}}}$ is weakly convergent to $\mu_{W_{\infty}}$. Moreover, $W_{n_{k}} \subset W_{\infty}$ for $k \gg 0$, and the irreducible component $Z_{\infty}$ of $\psi^{-1}\left(W_{\infty}\right)$ containing $Z_{n_{k}}, k \gg 0$ is a non-factor special subvariety of Shimura type of $S$ and $\psi$-dominant over $W_{\infty}$.

1.5. Strategy of the proof. The method we use to prove Theorem 1.11 is from ergodic theory, due to Ratner ([Rat91-1], [Rat91-2]) Mozes-Shah [MS95] and Dani-Margulis [DM91]. And we deduce Theorem 1.7 from Theorem 1.11 and the definability of period maps. More precisely:

1. Assuming the Hodge variety $\operatorname{Hod}_{\Gamma}(S, \mathbb{V})$ contains one non-factor Shimura type special subvariety, then these non-factor Shimura type special subvarieties will equidistribute in $\operatorname{Hod}_{\Gamma}(S, \mathbb{V})$. We will follow the strategies used by Clozel and Ullmo in [CU05-1] and Ullmo in [Ull07]. But there are two main differences that we want to address:

(a) for a non-factor special subvariety $W$ of $\operatorname{Hod}_{\Gamma}(S, \mathbb{V})$ associated to a Hodge subdatum $\left(\boldsymbol{H}, \mathcal{D}_{\boldsymbol{H}}\right)$ of $(\boldsymbol{G}, \mathcal{D})$ with $\boldsymbol{G}$ semisimple of adjoint type, we need to show that the centralizer $\mathbf{Z}_{\boldsymbol{G}}\left(\boldsymbol{H}^{\mathrm{der}}\right)(\mathbb{R})$ of $\boldsymbol{H}^{\text {der }}$ in $\boldsymbol{G}$ is contained in $M_{h}$, the isotropy subgroup in $G(\mathbb{R})$ of a Hodge generic point $h \in \mathcal{D}_{H}$. Ullmo's method doesn't apply here since the Hodge datum $(\boldsymbol{G}, \mathcal{D})$ is in general not a Shimura datum. We give a Hodge-theoretic proof of this result (Proposition 4.4), which works in all cases.

(b) we need to show the limit of a sequence of non-factor Shimura type special subvarieties is again of Shimura type. This is quite easy in the classical case as $(G, \mathcal{D})$ is itself of Shimura type.

2. We need to know that there are only finitely many components in the preimage $\psi^{-1}(W)$ of a special subvariety $W$ of $\operatorname{Hod}_{\Gamma}(S, \mathbb{V})$ under the period map. This follows from the recent result of Bakker, Klingler and Tsimerman [BKT18] on the definability of period map $\psi$.

As for the organization of this paper, in Section 2 we provide a recollection of the ergodic results that we need. In Section 3 we recall the general definitions of a Hodge datum and of a Hodge variety and review the definability of the period map. In Section 4, we discuss the equidistribution of non-factor special subvarieties. Section 5 and 6 give the proof of the main results.

Notations. An algebraic group will be denoted by boldface (or blackboard bold) letters (e. g. $\mathbb{S}, \boldsymbol{G}, \boldsymbol{H}, \cdots$ ) and a Lie group will be denoted by usual letters (e. g. $G, H, \cdots$ ).

Let $H$ be an algebraic group. 
- The adjoint group and derived subgroup of $\boldsymbol{H}$ are denoted by $H^{\text {ad }}$ and $H^{\text {der }}$ respectively; the centralizer (resp. normalizer) of a subgroup $\boldsymbol{H}$ in an algebraic group $G$ is denoted by $\mathbf{Z}_{\boldsymbol{G}}(\boldsymbol{H})\left(\right.$ resp. $\left.\mathbf{N}_{\boldsymbol{G}}(\boldsymbol{H})\right)$;

- If $\boldsymbol{H}$ is defined over $\mathbb{R}$, we denote $\boldsymbol{H}(\mathbb{R})^{+}$the identity component of $\boldsymbol{H}(\mathbb{R})$ for the real topology and $\boldsymbol{H}(\mathbb{R})_{+}$the preimage of $\boldsymbol{H}^{\text {ad }}(\mathbb{R})^{+}$under the adjoint homomorphism ad $: \boldsymbol{H} \rightarrow \boldsymbol{H}^{\text {ad }}$;

- If $\boldsymbol{H}$ is connected semisimple and defined over a filed $k$, then $\boldsymbol{H}$ is the almost direct product of its minimal nonfinite normal $k$-subgroups $\boldsymbol{H}_{1}, \cdots, \boldsymbol{H}_{r}$ (cf. [Mil17] theorem 21.51). If $\boldsymbol{H}$ is adjoint or simply connected, the product is direct. By abuse of language, the $H_{i}$ are called $k$-simple factors of $\boldsymbol{H}$.

Acknowledgement. I wish to record my indebtedness to my supervisor Bruno Klingler during the preparation of this paper. I had numerous discussions with him, which have influenced this paper.

\section{Some ergodic results à la Ratner, Mozes and Shah}

In this section, we will review some results form ergodic theory ([Rat91-1], [Rat91-2] and [MS95]) that will be used later. We follow the same terminologies as defined in [CU05-1], [CU05-2] and [Ull07].

\subsection{Algebraic groups of type $\mathcal{K}$ and Lie subgroups of type $\mathcal{H}$.}

Definition 2.1. A connected linear $\mathbb{Q}$-algebraic group $H$ is said to be of type $\mathcal{K}$ if its radical is unipotent, and $\boldsymbol{H}^{\mathrm{ss}}:=\boldsymbol{H} / \mathrm{R}_{\mathrm{u}}(\boldsymbol{H})$ is of non-compact type: that is, none of its $\mathbb{Q}$-simple factors are $\mathbb{R}$-anisotropic. Here $\mathrm{R}_{\mathrm{u}}(\boldsymbol{H})$ denotes the unipotent radical of $\boldsymbol{H}$.

Let $G$ be a connected semisimple $\mathbb{Q}$-algebraic group and $G=G(\mathbb{R})^{+}$be the associated connected Lie group. Let $\Gamma$ be an arithmetic lattice of $G$ and $\Omega$ be the homogeneous space $\Gamma \backslash G$ on which $G$ acts by right translations. Let $\mathcal{P}(\Omega)$ denote the set of Borel probability measures on $\Omega$ equipped with the weak-* topology.

Definition 2.2. (cf. [CU05-1] Section 2). Let $H$ be a connected closed Lie subgroup of $G$. Then $H$ is said to be of type $\mathcal{H}$ if

(i) $H \cap \Gamma$ is a lattice of $H$. In particular, the orbit $\Gamma \backslash \Gamma H$ of $\Gamma e \in \Omega$ under $H$ is closed in $\Omega$ ([Rat91-1], Proposition 1.4). We denote by $\mu_{H} \in \mathcal{P}(\Omega)$ the unique $H$-invariant Borel probability measure supported on $\Gamma \backslash \Gamma H$.

(ii) The subgroup $L(H) \subset H$ generated by the one-parameter unipotent subgroups of $G$ contained in $H$ acts ergodically on $\Gamma \backslash \Gamma H$ with respect to the measure $\mu_{H}$.

Note that the definition of an algebraic group being type $\mathcal{K}$ is intrinsic, while the notion of type $\mathcal{H}$ is for a subgroup of a given group. The relation between type $\mathcal{K}$ algebraic subgroups 
of $G$ and type $\mathcal{H}$ closed Lie subgroups of $G$ is given by the following lemma, proven as Lemmas 2.1, 2.2 and 2.3 in [CU05-1].

Lemma 2.3. Let $G$ be a connected semisimple $\mathbb{Q}$-algebraic subgroup of type $\mathcal{K}$.

(1) If $\boldsymbol{H}$ is a connected semisimple $\mathbb{Q}$-subgroup of $\boldsymbol{G}$ of type $\mathcal{K}$, then $H:=\boldsymbol{H}(\mathbb{R})^{+}$is a closed Lie subgroup of $G$ of type $\mathcal{H}$.

(2) If $H$ is a connected Lie subgroup of $G$ of type $\mathcal{H}$, then there exists a connected $\mathbb{Q}$-algebraic subgroup $\boldsymbol{H}$ of $\boldsymbol{G}$ of type $\mathcal{K}$ such that $H:=H(\mathbb{R})^{+}$.

Remark 2.4. The $\mathbb{Q}$-algebraic subgroup $H$ in part (2) of Lemma 2.3 is constructed as the $\mathbb{Q}$-Zariski closure of $H$ in $G$.

2.2. A theorem of Mozes and Shah. Let $\mathcal{Q}(\Omega)$ be the subset of $\mathcal{P}(\Omega)$ consisting of all the $H$-invariant Borel probability measures $\mu_{H}$ associated to type $\mathcal{H}$ closed connected Lie subgroups $H$ of $G$.

Theorem 2.5 (Mozes-Shah [MS95], Theorem 1.1 and Corollary 1.4).

(1) $\mathcal{Q}(\Omega)$ is a compact subset of $\mathcal{P}(\Omega)$.

(2) If $\left(\mu_{n}\right)$ is a sequence in $Q(\Omega)$ that weakly converges to $\mu \in Q(\Omega)$, then the supports $\operatorname{supp}\left(\mu_{n}\right)$ are contained in $\operatorname{supp}(\mu)$ for $n$ big enough.

\section{Hodge VARIETIES AND DEFINABILITY OF PERIOD MAPS}

In this section we recall the definition of special subvarieties of a Hodge variety and give a brief review of the definability of period maps. The main references for this section are [GGK12], [CMP17], [K17], and [BKT18].

3.1. Hodge data and Hodge varieties. Let $\mathbb{S}:=\operatorname{Res}_{\mathbb{C} / \mathbb{R}} \mathbb{G}_{m, \mathbb{C}}$ denote the Deligne torus. It is the Tannaka dual group of the category of real Hodge structures. The inclusion $\mathbb{R}^{\times} \hookrightarrow \mathbb{C}^{\times}$ corresponds to an inclusion of real algebraic groups $w: \mathbb{G}_{m, \mathbb{R}} \hookrightarrow \mathbb{S}$.

\section{Definition 3.1.}

(i) A Hodge datum is a pair $(\boldsymbol{G}, \mathcal{D})$ consisting of a connected $\mathbb{Q}$-reductive group and a $\boldsymbol{G}(\mathbb{R})$-conjugacy class $\mathcal{D}$ of some homomorphism $h \in \operatorname{Hom}\left(\mathbb{S}, \boldsymbol{G}_{\mathbb{R}}\right)$ satisfying the following conditions:

HD 0: the weight homomorphism $w_{h}:=h \circ w: \mathbb{G}_{m, \mathbb{R}} \rightarrow \boldsymbol{G}_{\mathbb{R}}$ is a cocharacter of the center of $\boldsymbol{G}_{\mathbb{R}}$ and is defined over $\mathbb{Q}$;

HD 1: the involution $\operatorname{Int}(h(\sqrt{-1}))$ is a Cartan involution of the adjoint group $\boldsymbol{G}_{\mathbb{R}}^{\text {ad }}$.

(ii) Let $(\boldsymbol{G}, \mathcal{D})$ be a Hodge datum and $\mathcal{D}^{+}$be a connected component of $\mathcal{D}$. The pair $\left(\boldsymbol{G}, \mathcal{D}^{+}\right)$is then called a connected Hodge datum.

(iii) A (connected) Hodge datum $(\boldsymbol{G}, \mathcal{D})\left(\operatorname{resp} .\left(\boldsymbol{G}, \mathcal{D}^{+}\right)\right)$is said to be of Shimura type if it satisfies two more conditions: 
HD 2: the Hodge structure induced on the Lie algebra $\operatorname{Lie}\left(\boldsymbol{G}_{\mathbb{R}}\right)$ by Ad $\circ h$ is of type

$$
\{(-1,1),(0,0),(1,-1)\} .
$$

HD 3: $G^{\text {ad }}$ has no $\mathbb{Q}$-factor on which the projection of $h$ is trivial. By the presence of axioms HD 1 and HD 2, this is equivalent to say that $G^{\text {ad }}$ is of non-compact type.

Remark 3.2.

(1) For $(G, \mathcal{D})$ a Hodge datum, there exists a unique structure of complex manifold on $\mathcal{D}$ such that for some (any) faithful (finite dimensional, algebraic) representation of $G$, the associated family of Hodge structures on $\mathcal{D}$ varies holomorphically (cf. [Mil05] Theorem 2.14).

(2) Any discrete subgroup $\Gamma$ of $G(\mathbb{Q})_{+}:=G(\mathbb{Q}) \cap G(\mathbb{R})_{+}{ }^{3}$ acts properly discontinuously on $\mathcal{D}^{+}$, so that $\Gamma \backslash \mathcal{D}^{+}$is a complex analytic space with at most finite quotient singularities (cf. [CMP17] Section 16.3).

\section{Definition 3.3.}

(i) Let $(\boldsymbol{G}, \mathcal{D})$ be a Hodge datum and $K$ be a compact open subgroup of $\boldsymbol{G}\left(\mathbb{A}_{f}\right)$ where $\mathbb{A}_{f}$ is the ring of finite adèles of $\mathbb{Q}$. The Hodge variety is defined as

$$
\operatorname{Hod}_{K}(\boldsymbol{G}, \mathcal{D}):=\boldsymbol{G}(\mathbb{Q}) \backslash \mathcal{D} \times \boldsymbol{G}\left(\mathbb{A}_{f}\right) / K,
$$

where $\boldsymbol{G}(\mathbb{Q})$ acts diagonally on $\mathcal{D}$ and $\boldsymbol{G}\left(\mathbb{A}_{f}\right)$ on the left and $K$ acts on $\boldsymbol{G}\left(\mathbb{A}_{f}\right)$ on the right.

(ii) Let $\left(\boldsymbol{G}, \mathcal{D}^{+}\right)$be a connected Hodge datum. A connected Hodge variety associated to $\left(G, \mathcal{D}^{+}\right)$is defined as the quotient $\Gamma \backslash \mathcal{D}^{+}$for an arithmetic subgroup $\Gamma$ of $G(\mathbb{Q})_{+}$.

Remark 3.4.

(1) As in the case of Shimura varieties, every connected Hodge variety is a connected component of a Hodge variety and vice versa (cf. [CMP17] Lemma 16.3.8). If $K$ (resp. $\Gamma$ ) is chosen sufficiently small, then $\operatorname{Hod}_{K}(\boldsymbol{G}, \mathcal{D})\left(\right.$ resp. $\left.\Gamma \backslash \mathcal{D}^{+}\right)$is a complex manifold and the map $\mathcal{D} \rightarrow \operatorname{Hod}_{K}(\boldsymbol{G}, \mathcal{D})$ (resp. $\mathcal{D}^{+} \rightarrow \Gamma \backslash \mathcal{D}^{+}$) is unramified.

(2) In general, the Hodge variety $\operatorname{Hod}_{K}(\boldsymbol{G}, \mathcal{D})\left(\right.$ resp. connected Hodge variety $\left.\Gamma \backslash \mathcal{D}^{+}\right)$ does not admit any algebraic structure (see [GRT14] Theorem 1.4).

We will only consider connected Hodge data and connected Hodge varieties in this paper.

Definition 3.5. A Hodge morphism of connected Hodge data $\left(\boldsymbol{G}, \mathcal{D}^{+}\right) \rightarrow\left(\boldsymbol{G}^{\prime}, \mathcal{D}^{\prime+}\right)$ is a homomorphism of $\mathbb{Q}$-algebraic groups $\varphi: G \rightarrow G^{\prime}$ which induces a map $\mathcal{D}^{+} \rightarrow \mathcal{D}^{\prime+}, h \mapsto \varphi \circ h$. A Hodge morphism of connected Hodge varieties is a morphism of varieties induced by a morphism of connected Hodge data.

\footnotetext{
${ }^{3}$ Recall that $G(\mathbb{R})_{+}$is the stabilizer of $\mathcal{D}^{+}$in $G(\mathbb{R})$.
} 
Remark 3.6. Let $h \in \mathcal{D}^{+}$and let $\mathcal{D}^{\text {ad, }+}$ be the $G^{\text {ad }}(\mathbb{R})^{+}$-conjugacy class of the composition $h^{\text {ad }}: \mathbb{S} \rightarrow G_{\mathbb{R}} \rightarrow G_{\mathbb{R}}^{\text {ad }}$. Then $\mathcal{D}^{+} \cong \mathcal{D}^{\text {ad, }+}$ and we have a morphism of connected Hodge data $\left(G, \mathcal{D}^{+}\right) \rightarrow\left(G^{\text {ad }}, \mathcal{D}^{\text {ad, },+}\right)$.

3.2. Special subvarieties of a connected Hodge variety. Let $\left(G, \mathcal{D}^{+}\right)$be a connected Hodge datum and let $Y$ be a connected Hodge variety associated to $\left(G, \mathcal{D}^{+}\right)$.

Definition 3.7. The image of any Hodge morphism $W \rightarrow Y$ between connected Hodge varieties is called a special subvariety of $Y$. It is said to be of Shimura type if the connected Hodge datum corresponds to $W$ is a Shimura datum.

For any special subvariety of $Y$, the Hodge morphism in Definition 3.7 can be chosen such that the underlying homomorphism of algebraic groups is injective. Hence any special subvariety of $Y$ can be regarded as given by a Hodge subdatum.

3.3. Special subvarieties associated to a $\mathbb{Z}-$ VHS. Let $\mathbb{V}$ be a $\mathbb{Z}-\mathrm{VHS}$ on a smooth irreducible complex quasi-projective variety $S$. Let $G$ be its generic Mumford-Tate group. Fix a Hodge generic point $o \in S$. The Hodge structure on the fiber $\mathbb{V}_{\mathbb{Q}, o} \cong V^{4}$ induces a morphism of $\mathbb{R}$-algebraic groups $h_{o}: \mathbb{S} \rightarrow \boldsymbol{G}_{\mathbb{R}}$. Let $\mathcal{D}$ be the $\boldsymbol{G}(\mathbb{R})$-conjugacy class of $h_{o}$ and let $\mathcal{D}^{+}$be a connected component of $\mathcal{D}$ containing $h_{o}$. Then we get a connected Hodge datum $\left(G, \mathcal{D}^{+}\right)$.

Let $\Gamma$ be a neat arithmetic lattice of $G(\mathbb{R})_{+}$, the stabilizer of $\mathcal{D}^{+}$in $G(\mathbb{R})$. After passing to a finite étale covering of $S$, we may assume that $\Gamma$ contains the monodromy group, namely the image of $\pi_{1}(S)$ in $\mathbf{G L}\left(\mathbb{V}_{\mathbb{Z}, o}\right)$. We denote by $\operatorname{Hod}_{\Gamma}^{\circ}(S, \mathbb{V})$ the connected Hodge variety $\Gamma \backslash \mathcal{D}^{+}$ associated to $(S, \mathbb{V})$. This is an arithmetic quotient $\Gamma \backslash \boldsymbol{G}(\mathbb{R})_{+} / M_{o}$ in the sense of [BKT18]. Here $M_{o}$ is the intersection of the isotropy subgroup of $h_{o}$ in $\boldsymbol{G}(\mathbb{R})$ with $\boldsymbol{G}(\mathbb{R})_{+}$, whose image in $G^{a d}(\mathbb{R})^{+}$turns out to be compact. And we have the period map:

$$
\psi: S \rightarrow \operatorname{Hod}_{\Gamma}^{\circ}(S, \mathbb{V}),
$$

which is holomorphic, locally liftable and all the local liftings are horizontal.

Let $K=\mathbf{Z}_{\boldsymbol{G}(\mathbb{R})}\left(h_{o}(\sqrt{-1})\right) \cap \boldsymbol{G}(\mathbb{R})_{+}$. Then $M_{o} \subset K$. And we have a canonical projection

$$
\omega: \mathcal{D}^{+}=G(\mathbb{R})_{+} / M_{o} \longrightarrow G(\mathbb{R})_{+} / K .
$$

Let $\mathfrak{g}:=\operatorname{Lie}\left(\boldsymbol{G}_{\mathbb{R}}\right), \mathfrak{k}:=\operatorname{Lie}(K)$, and $\mathfrak{m}:=\operatorname{Lie}\left(M_{o}\right)$. Then $\mathfrak{g}$ carries a weight 0 Hodge structure

$$
\mathfrak{g}_{\mathbb{C}}=\bigoplus \mathfrak{g}^{-j, j}
$$

\footnotetext{
${ }^{4}$ The pullback of the local system $\mathbb{V}_{\mathbb{Q}}$ to the topological universal cover $\hat{S}$ of $S$ is constant, hence isomorphic to $\hat{S} \times V$ for some finite dimensional $\mathbb{Q}$-vector space $V$.
} 
polarized by minus the Killing form of $\mathfrak{g}$. And by the axiom (HD 1), we have a Cartan decomposition

$$
\mathfrak{g}=\mathfrak{k} \oplus \mathfrak{p} .
$$

Let $T_{\omega}$ (resp. $T_{\omega}^{\perp}$ ) be the subbundle of the tangent bundle $T_{\mathcal{D}^{+}}$of $\mathcal{D}^{+}$associated to the adjoint representation of $M_{o}$ on $\mathfrak{k} / \mathfrak{m}$ (resp. $\left.\mathfrak{p}\right)$. We then have a canonical splitting

$$
T_{\mathcal{D}^{+}}=T_{\omega} \oplus T_{\omega}^{\perp} .
$$

The subbundle $T_{\omega}$ is holomorphic as the fibers of $\omega$ are complex submanifolds of $\mathcal{D}^{+}$, while $T_{\omega}^{\perp}$ in general admits no complex structure. However, there is a holomorphic subbundle $T_{\mathcal{D}^{+}}^{h}$ contained in the complexification $T_{\omega}^{\perp} \otimes \mathbb{C}$, namely the subbundle associated to the adjoint representation of $M_{o}$ on $g^{-1,1}$ and we call it the holomorphic horizontal tangent bundle. When we say the period map $\psi$ is horizontal, we mean

$$
d \hat{\psi}\left(T_{\hat{S}}\right) \subset \hat{\psi}^{*} T_{\mathcal{D}^{+}}^{h},
$$

where $\hat{S}$ is the topological universal cover of $S$ and $\hat{\psi}$ is the lifting of $\psi$ to $\hat{S}$.

Given an irreducible algebraic subvariety $Z$ of $S$, let $\tilde{Z} \rightarrow Z$ be its normalization and $\tilde{Z}^{\text {sm }}$ be the smooth locus of $\tilde{Z}$. Let $u: \tilde{Z}^{\text {sm }} \hookrightarrow \tilde{Z} \rightarrow Z \hookrightarrow S$ be the composition. Then the local system $u^{*} \mathbb{V}$ on $\tilde{Z}^{\text {sm }}$ is a $\mathbb{Z}$-VHS, and we denote its generic Mumford-Tate group by $G_{Z}$. Let

$$
\tilde{\psi}_{Z}: \tilde{Z}^{\mathrm{sm}} \rightarrow \operatorname{Hod}_{\Gamma_{Z}}^{\circ}\left(\tilde{Z}^{\mathrm{sm}}, u^{*} \mathbb{V}\right)=\Gamma_{Z} \backslash \mathcal{D}_{Z}^{+}
$$

be the associated period map, where $\Gamma_{Z}=\Gamma \cap G_{Z}(\mathbb{Q})$, we then have a commutative diagram

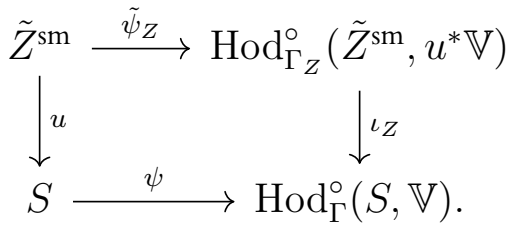

Notice that the restriction of the period map $\psi$ to the smooth locus of $Z$ factors through the special subvariety $\operatorname{Im}\left(\iota_{Z}\right)$ of $\operatorname{Hod}_{\Gamma}^{\circ}(S, \mathbb{V})$ and every complex analytic irreducible component of the preimage $\psi^{-1}\left(\operatorname{Im}\left(\iota_{Z}\right)\right)$ is a special subvariety of $S$ for $\mathbb{V}$. Conversely, if $Z$ is a special subvariety for $(S, \mathbb{V})$, then it follows readily from the Definition 1.1 that $Z$ is a complex analytic irreducible component of the preimage $\psi^{-1}\left(\operatorname{Im}\left(\iota_{Z}\right)\right)$. We thus prove the following lemma (the last assertion is obvious):

Lemma 3.8. The special subvarieties for $(S, \mathbb{V})$ are precisely the preimages of the special subvarieties for $\operatorname{Hod}_{\Gamma}^{\circ}(S, \mathbb{V})$. Moreover, the preimages of special points ${ }^{5}$ in $\operatorname{Hod}_{\Gamma}^{\circ}(S, \mathbb{V})$ are $C M$ points for $(S, \mathbb{V})$.

${ }^{5}$ Zero-dimensional special subvarieties (namely, special points) for a Hodge variety are precisely the CM points (cf. [CMP17] examples 16.3.7) 
Remark 3.9. It can be shown that the set of $\mathrm{CM}$ points is dense in $\operatorname{Hod}_{\Gamma}^{\circ}(S, \mathbb{V})$ (cf. [CMP17] Corollary 17.1.5). However, there is no guarantee for the image of the period map $\psi(S)$ to contain even one CM point.

Definition 3.10. Let $Z$ be an irreducible algebraic subvariety of $S$. The algebraic monodromy group $\boldsymbol{H}_{Z}$ of $Z$ for $\mathbb{V}$ is defined to be the Zariski closure in $\mathbf{G L}(V)$ of the monodromy group of the local system $u^{*} \mathbb{V}_{\mathbb{Z}}$ on $\tilde{Z}^{\text {sm }}$.

3.4. Definability of period maps and algebraicity of special subvarieties. Although the period map $\psi$ is transcendental, Bakker, Klingler and Tsimerman [BKT18] showed that it has moderate geometry in the sense of tame topology. For a reference to the notions of tame topology and definability in some o-minimal structure (for instance $\mathbb{R}_{\mathrm{alg}}, \mathbb{R}_{\mathrm{an}}, \mathbb{R}_{\mathrm{an}, \exp }, \cdots$ ), see $[\mathrm{vdD} 98]$.

Theorem 3.11 (Bakker, Klingler and Tsimerman).

(1) There is a natural $\mathbb{R}_{\mathrm{alg}}$-definable manifold structure on the connected Hodge variety $\operatorname{Hod}_{\Gamma}^{\circ}(S, \mathbb{V})$.

(2) With respect to the $\mathbb{R}_{\mathrm{an}, \exp }$-definable manifold structure extending the $\mathbb{R}_{\mathrm{alg}}$-definable manifold structure on $S\left(\right.$ resp. on $\operatorname{Hod}_{\Gamma}^{\circ}(S, \mathbb{V})$ ) coming from its complex algebraic structure (resp. defined in part (1)), the period map $\psi: S \rightarrow \operatorname{Hod}_{\Gamma}^{\circ}(S, \mathbb{V})$ is $\mathbb{R}_{\text {an,exp }}$-definable.

(3) For any special subvariety $Y$ of $\operatorname{Hod}_{\Gamma}^{\circ}(S, \mathbb{V})$, the preimage $\psi^{-1}(Y)$ is an algebraic subvariety of $S$. In particular, $\psi^{-1}(Y)$ has only finitely many irreducible components.

Proof. Part (1) is Theorem 1.1 (1) in [BKT18], part (2) is Theorem 1.3 in [BKT18] and part (3) is Theorem 1.6 in [BKT18] .

3.5. The structure theorem for period maps. For later use, we need a structure theorem for period maps.

Let $H$ be the algebraic monodromy group of $S$ for $\mathbb{V}$. It follows from [An92] Theorem 5.1 that $H$ is a normal subgroup of derived subgroup $G^{\text {der }}$ of the generic Mumford-Tate group $G$. As $G^{\text {der }}$ is semisimple, there exists a normal subgroup $F$ of $G^{\text {der }}$ such that $G^{\text {der }}$ is an almost direct product of $\boldsymbol{H}$ and $\boldsymbol{F}$. Let $\boldsymbol{H}^{\mathrm{nc}}$ and $\boldsymbol{H}^{\mathrm{c}}$ be the non-compact and compact part of $H$ respectively. Then we will have an isogeny of $\mathbb{Q}$-reductive groups:

$$
\boldsymbol{H}^{\mathrm{nc}} \times \boldsymbol{H}^{\mathrm{c}} \times \boldsymbol{F} \rightarrow \boldsymbol{G}^{\mathrm{der}}
$$

which induces a surjective holomorphic map with finite fibers between Mumford-Tate domains:

$$
\mathcal{D}_{\boldsymbol{H}^{\mathrm{nc}}}^{+} \times \mathcal{D}_{\boldsymbol{H}^{\mathrm{c}}}^{+} \times \mathcal{D}_{\boldsymbol{F}}^{+} \rightarrow \mathcal{D}^{+} .
$$

Here for a $\mathbb{Q}$-algebraic subgroup $G^{\prime}$ of $G$, we write $\mathcal{D}_{G^{\prime}}$ for the $G^{\prime}(\mathbb{R})$-orbit in $\mathcal{D}$ of a fixed lifting in $\mathcal{D}$ of the image $\psi(o) \in \operatorname{Hod}_{\Gamma}^{\circ}(S, \mathbb{V})$ and $\mathcal{D}_{G^{\prime}}^{+}$the connected component of $\mathcal{D}_{G^{\prime}}$ containing the lifting. 
Theorem 3.12. Let $\mathbb{V}$ be a $\mathbb{Z}-V H S$ on a smooth irreducible complex quasi-projective variety $S$. Then its associated period map $\psi: S \rightarrow \operatorname{Hod}_{\Gamma}^{\circ}(S, \mathbb{V})$ factors as:

$$
\psi=\left(\psi_{n c}, \psi_{c}, \psi_{f}\right): S \longrightarrow \Gamma^{n c} \backslash \mathcal{D}_{\boldsymbol{H}^{n c}}^{+} \times \Gamma^{c} \backslash \mathcal{D}_{\boldsymbol{H}^{c}}^{+} \times \mathcal{D}_{\boldsymbol{F}}^{+},
$$

where $\Gamma^{n c}:=\Gamma \cap \boldsymbol{H}^{n c}(\mathbb{Q})$ and $\Gamma^{c}:=\Gamma \cap \boldsymbol{H}^{c}(\mathbb{Q})$. Moreover,

(1) the component $\psi_{f}$ is constant; correspondingly, the $\mathbb{Z}-V H S \mathbb{V}$ is a direct sum of a sub-VHS whose generic Mumford-Tate group is the whole group $G$ and a $(n)$ (iso-)trivial one. Let $e_{3}$ be image of $S$ under $\psi_{f}$.

(2) for any point $x \in \mathcal{D}_{\boldsymbol{H}^{c}}^{+} \subset \mathcal{D}^{+}$, we have $T_{\mathcal{D}_{\boldsymbol{H}^{c}}^{+}, x} \subset T_{\omega, x}$. As a consequence, for any $e_{1} \in \psi_{n c}(S)$, the image $\psi(S)$ intersects $e_{1} \times \Gamma^{c} \backslash \mathcal{D}_{\boldsymbol{H}^{c}}^{+} \times e_{3}$ in finitely many point.

(3) the number of points of the intersections of $\psi(S)$ with $e_{1} \times \Gamma^{c} \backslash \mathcal{D}_{\boldsymbol{H}^{c}}^{+} \times e_{3}$ is uniformly bounded as $e_{1}$ varies in $\psi_{n c}(S)$.

Proof. For the proof of (1) and (2), see Chapter 15 of [CMP17]. Let us prove (3). Since $\Gamma^{\mathrm{c}} \backslash \mathcal{D}_{\boldsymbol{H}^{\mathrm{c}}}^{+}$is compact, the projection

$$
\Gamma^{\mathrm{nc}} \backslash \mathcal{D}_{\boldsymbol{H}^{\mathrm{nc}}}^{+} \times \Gamma^{\mathrm{c}} \backslash \mathcal{D}_{\boldsymbol{H}^{\mathrm{c}}}^{+} \longrightarrow \Gamma^{\mathrm{nc}} \backslash \mathcal{D}_{\boldsymbol{H}^{\mathrm{nc}}}^{+}
$$

is $\mathbb{R}_{\mathrm{an}}$-definable. Since the period map $\psi$ is $\mathbb{R}_{\mathrm{an}, \exp }$-definable by Theorem 3.11 , the map

$$
\psi(S) \longrightarrow \psi_{n c}(S)
$$

is $\mathbb{R}_{\mathrm{an} \text {,exp }}$-definable. Since each fiber of this map is finite by (2), the uniformly boundedness then follows from the finiteness lemma (the Lemma 1.7 in Chapter 3, Section 1 of [vdD98]).

\section{NON-FACTOR SPECIAL SUBVARIETIES}

4.1. In this section, we introduce the notion of non-factor special subvarieties (Definition 4.1). This is a natural definition from the equidistribution point of view: as explained in [Ull07], for a sequence $\left(Y_{n}\right)$ of special subvarieties of $\operatorname{Hod}_{\Gamma}^{\circ}(S, \mathbb{V})$, we cannot expect in general that the associated sequence of Borel probability measures $\mu_{n}=\mu_{Y_{n}}$ on $\operatorname{Hod}_{\Gamma}^{\circ}(S, \mathbb{V})$ with support $Y_{n}$ weakly converges. For example, if $\left(Y_{n}\right)$ is a sequence of special points in $\operatorname{Hod}_{\Gamma}^{\circ}(S, \mathbb{V})$, then $\mu_{n}$ is just the Dirac measure supported at the point $Y_{n}$. Such a sequence can converge to a non special point or may tend to $\infty$. Even for positive dimensional special subvarieties the same problem may occur. Start with a special subvariety of $\operatorname{Hod}_{\Gamma}^{\circ}(S, \mathbb{V})$ of the form $Y \times Y^{\prime}$ for two special subvarieties $Y$ and $Y^{\prime}$. Let $\left(y_{n}\right)$ be a sequence of special point of $Y^{\prime}$ and $Y_{n}=Y \times\left\{y_{n}\right\}$, then there is no hope of proving the weak convergence of $\mu_{n}$.

Definition 4.1 (cf. [Ullo7]).

(i) Let $Y$ be a connected Hodge variety. A special subvariety $W$ of $Y$ is called non-factor if there exists no finite morphism of connected Hodge varieties:

$$
W_{1} \times W_{2} \rightarrow Y
$$


with $W_{2}$ having positive dimension, such that $W$ is the image of $W_{1} \times\{x\}$ in $Y$ for any (necessary special) point $x$ of $W_{2}$.

(ii) Let $\mathbb{V}$ be a $\mathbb{Z}$-VHS on a smooth irreducible complex quasi-projective variety $S$ and let $\operatorname{Hod}_{\Gamma}^{\circ}(S, \mathbb{V})$ be the associated connected Hodge variety. A special subvariety $Z$ for $(S, \mathbb{V})$ is called non-factor if $\Gamma_{Z} \backslash \mathcal{D}_{Z}^{+}$is a non-factor special subvariety of $\operatorname{Hod}_{\Gamma}^{\circ}(S, \mathbb{V})$.

Remark 4.2. Note that any Hodge variety $Y$ itself is non-factor. Assume that $Y$ is of positive dimensional. For a special point $x \in Y$, the projection

$$
\{x\} \times Y \rightarrow Y
$$

is a finite morphism. This shows that special points are not non-factor special subvarieties of connected Hodge variety.

Remark 4.3. A special subvariety which contains a non-factor special subvariety is automatically non-factor. And $W$ is a non-factor special subvariety of $Y$ if and only if $W^{\text {ad }}$ is a non-factor special subvariety of $Y^{\text {ad }}$.

There is a useful group-theoretic characterization of non-factor special subvarieties for a variation of Hodge structure.

Let $\mathbb{V}$ be a $\mathbb{Z}$-VHS on a smooth irreducible complex quasi-projective variety $S$ with associated Hodge datum $\left(G, \mathcal{D}^{+}\right)$. We assume that $G$ is semisimple of adjoint type. Let $Z$ be a special subvariety for $(S, \mathbb{V})$ with associated Hodge subdatum $\left(\boldsymbol{G}_{Z}, \mathcal{D}_{Z}^{+}\right) \hookrightarrow\left(\boldsymbol{G}, \mathcal{D}^{+}\right)$. Let $h$ be a Hodge generic point in $\mathcal{D}_{Z}^{+}$and denote its isotropy group in $G(\mathbb{R})$ by $M_{h}$. Note that $M_{h}$ is a compact subgroup of $\boldsymbol{G}(\mathbb{R})$.

Proposition 4.4. If $Z$ is a non-factor special subvariety for $(S, \mathbb{V})$, then the centralizer $\mathbf{Z}_{G}\left(G_{Z}^{\text {der }}\right)(\mathbb{R})$ is contained in $M_{h}$.

Proof. Let $\mathfrak{g}:=\operatorname{Lie}(\boldsymbol{G}), \mathfrak{h}:=\operatorname{Lie}\left(\boldsymbol{G}_{Z}^{\text {der }}\right)$ and $\mathfrak{c}:=\operatorname{Lie}\left(\mathbf{Z}_{G}\left(\boldsymbol{G}_{Z}^{\text {der }}\right)\right)$. Since $\boldsymbol{G}_{Z}^{\text {der }}$ is semisimple, the Lie algebra $\mathfrak{g}$ decomposes as a $G_{Z}^{\mathrm{der}}$-module as follows:

$$
\mathfrak{g}=\mathfrak{h} \oplus \mathfrak{c} \oplus \mathfrak{l} .
$$

where $\mathfrak{l}$ is the orthogonal complement of $\mathfrak{h} \oplus \mathfrak{c}$ with respect to the Killing form of $\mathfrak{g}$.

Notice that $\mathfrak{g}$ carries a natural weight 0 polarized rational Hodge structure and this defines a variation of Hodge structure $\mathbb{V}_{\mathfrak{g}}$ on $S$. Let $\boldsymbol{H}_{Z}$ be the algebraic monodromy group of $Z$ for $\mathbb{V}$. It follows from [An92] Theorem 5.1 that $\boldsymbol{H}_{Z}$ is a normal subgroup of $\boldsymbol{G}_{Z}^{\mathrm{der}}$. So the above decomposition (2) of $\mathfrak{g}$ induces a decomposition of the underlying local system $\mathbb{V}_{\mathfrak{g}}$ :

$$
\mathbb{V}_{\mathfrak{g}}=\mathbb{V}_{\mathfrak{h}} \oplus \mathbb{V}_{\mathfrak{c}} \oplus \mathbb{V}_{\mathfrak{l}}
$$

Let $G^{1}$ be the connected $\mathbb{Q}$-algebraic subgroup of $G$ with Lie algebra $\mathfrak{h} \oplus \mathfrak{c}$. Then $G^{1}$ is reductive. And it can be seen easily that $G^{1}$ is the connected $\mathbb{Q}$-subgroup of $G$ generated by $\boldsymbol{G}_{Z}$ and $\left(\mathbf{Z}_{G}\left(\boldsymbol{G}_{Z}^{\mathrm{der}}\right)\right)^{\circ}$. In fact, $\boldsymbol{G}^{1}$ is the identity component of the normalizer of $\boldsymbol{G}_{Z}^{\mathrm{der}}$ in $\boldsymbol{G}$. 
Let $\mathcal{D}^{1} \subset \mathcal{D}$ be the $G^{1}(\mathbb{R})$-orbit of $h \in \mathcal{D}_{Z}^{+}$. Then $\left(G^{1}, \mathcal{D}^{1}\right)$ is a Hodge subdatum. By (3), we have a decomposition of the connected Hodge subdatum $\left(G^{1}, \mathcal{D}^{1,+}\right)$ and a finite morphism

$$
\left.\left(\boldsymbol{G}^{1}, \mathcal{D}^{1,+}\right) \cong\left(\boldsymbol{G}_{Z}, \mathcal{D}_{Z}^{+}\right) \times\left(\mathbf{Z}_{\boldsymbol{G}}\left(\boldsymbol{G}_{Z}^{\text {der }}\right)\right)^{\circ}, \mathcal{D}^{2,+}\right) \rightarrow(\boldsymbol{G}, \mathcal{D}) .
$$

If $\mathbf{Z}_{G}\left(G_{Z}^{\text {der }}\right)(\mathbb{R})$ is not contained in $M_{h}$, then $\mathcal{D}^{2,+}$ is of positive dimensional and $\Gamma_{Z} \backslash \mathcal{D}_{Z}^{+}$is the image of a $\Gamma_{Z} \backslash \mathcal{D}_{Z}^{+} \times\left\{x_{2}\right\}$ in $\operatorname{Hod}_{\Gamma}^{\circ}(S, \mathbb{V})$. So $Z$ is not non-factor, which is a contradiction.

4.2. A description of special subvarieties. Note that any point $h \in \mathcal{D}^{+}$induces a projection map

$$
\begin{aligned}
\pi_{h}: \quad \Omega:=\Gamma \backslash \boldsymbol{G}(\mathbb{R})^{+} & \rightarrow \Gamma \backslash \mathcal{D}^{+}=\operatorname{Hod}_{\Gamma}^{\circ}(S, \mathbb{V}) \\
{[g] } & \mapsto \quad[g h] .
\end{aligned}
$$

Let $Z$ be a special subvariety of $S$. We denote by $\left(\boldsymbol{G}_{Z}, \mathcal{D}_{Z}^{+}\right)$the corresponding connected Hodge subdatum and $W$ the corresponding special subvariety of $\operatorname{Hod}_{\Gamma}^{\circ}(S, \mathbb{V})$. For $h \in \mathcal{D}_{Z}^{+}$, let $M_{h}:=\mathbf{Z}_{\boldsymbol{G}(\mathbb{R})}(h)$ be the stabilizer of $h$ in $\boldsymbol{G}(\mathbb{R})$. Then $M_{h}$ is a compact subgroup containing the center of $G_{Z}(\mathbb{R})_{+}$. Hence we have the following description of $W$ :

$$
\begin{aligned}
W & =\Gamma \backslash \Gamma \boldsymbol{G}_{Z}(\mathbb{R})_{+} h \\
& =\Gamma \backslash \Gamma \boldsymbol{G}_{Z}^{\text {der }}(\mathbb{R})^{+} h=\pi_{h}\left(\Gamma \backslash \Gamma \boldsymbol{G}_{Z}^{\text {der }}(\mathbb{R})^{+}\right) \\
& \cong \Gamma \backslash \Gamma \boldsymbol{G}_{Z}^{\text {der }}(\mathbb{R})^{+} M_{h} / M_{h} .
\end{aligned}
$$

Let $\mu_{Z}$ be the unique $G_{Z}^{\text {der }}(\mathbb{R})^{+}$-invariant Borel probability measure supported on $\Gamma \backslash \Gamma G_{Z}^{\operatorname{der}}(\mathbb{R})^{+}$ and $\mu_{W}:=\left(\pi_{h}\right)_{*} \mu_{Z}$. As there is a canonical $G_{Z}^{\text {der }}(\mathbb{R})^{+}$-invariant metric on $\mathcal{D}_{Z}^{+}$, the measure $\mu_{W}$ is the same as the the normalized measure induced from the Hermitian metric. In particular the probability measure $\mu_{M}$ is independent of the choice of $h \in \mathcal{D}_{Z}^{+}$.

Let $\gamma \in \Gamma, \boldsymbol{G}_{Z, \gamma}=\gamma \boldsymbol{G}_{Z} \gamma^{-1}, h_{\gamma}=\gamma \cdot h$ and $\mathcal{D}_{Z, \gamma}$ the $\boldsymbol{G}_{Z, \gamma}(\mathbb{R})$-conjugacy class of $h_{\gamma}$. We also have

$$
W=\pi_{h_{\gamma}}\left(\Gamma \backslash \Gamma G_{Z, \gamma}^{\text {der }}(\mathbb{R})^{+}\right) .
$$

Fixing a fundamental domain $\mathcal{F}$ for the action of $\Gamma$ on $\mathcal{D}^{+}$, we can thus choose $h \in \mathcal{F}$ in the description of $W$.

4.3. Non-factor special subvarieties and recurrence to compact sets. We keep the same notations as in the sections 4.1 and 4.2. The following theorem is a corollary of a deep result of Dani and Margulis on the quantitative recurrence to compact sets for unipotent flows on $\Omega=\Gamma \backslash \boldsymbol{G}(\mathbb{R})^{+}$([DM91] Theorem 2). It tells us that unipotent flows never send lattices off to infinity, which (in principle) allows us to argue "as if" $\Omega$ was compact when considering unipotent flows. This will be a key ingredient in our proof of the main theorem. It was used by Clozel and Ullmo (cf. [CU05-1] Lemma 4.5) and Ullmo [Ull07] in their proof of 
equidistribution of strongly (more generally, non-factor) special subvarieties in a Shimura variety and it is not difficult to adapt their arguments to our situation.

Theorem 4.5. There exists a compact subset $C$ of $\operatorname{Hod}_{\Gamma}^{\circ}(S, \mathbb{V})$ such that $\Gamma_{Z} \backslash \mathcal{D}_{Z}^{+} \cap C \neq \varnothing$ for any non-factor special subvariety $Z$ of Shimura type for $(S, \mathbb{V})$.

Proof. It follows easily from [DM91] Theorem 2, that there exists a compact subset $C^{\prime}$ of $\Omega$ such that for all unipotent one-parameter subgroup $U \subset G(\mathbb{R})^{+}$and $g \in G(\mathbb{R})^{+}$, if

$$
\Gamma \backslash \Gamma g U \cap C^{\prime}=\varnothing,
$$

then there exist a proper $\mathbb{Q}$-parabolic subgroup $\boldsymbol{P}^{\prime}$ of $G$ such that

$$
g U g^{-1} \subset \boldsymbol{P}^{\prime}(\mathbb{R}) \text {. }
$$

Let $V \subset \boldsymbol{G}(\mathbb{R})^{+}$be a compact neighborhood of the identity element $e \in \boldsymbol{G}(\mathbb{R})^{+}$. Then

$$
C^{\prime \prime}:=C^{\prime} V=\{c v \mid c \in C, v \in V\}
$$

is also a compact subset of $\Omega$. Fix a point $h_{0} \in \mathcal{D}^{+}$and let $C=\pi_{h_{0}}\left(C^{\prime \prime}\right)$. Then for any point $\alpha \in V$, we have

$$
\pi_{h_{\alpha}}\left(C^{\prime}\right) \subset C,
$$

where $h_{\alpha}=\alpha \cdot h_{0}$.

For $h \in \mathcal{D}_{Z}^{+}$, since $\boldsymbol{G}(\mathbb{Q})^{+}$is dense in $\boldsymbol{G}(\mathbb{R})^{+}$, there exists $\alpha \in V$ and $\gamma \in \boldsymbol{G}(\mathbb{Q})^{+}$such that $h=\gamma \alpha \cdot h_{0}$. We then have

$$
\begin{aligned}
\Gamma_{Z} \backslash \mathcal{D}_{Z}^{+} & =\Gamma \backslash \Gamma \boldsymbol{G}_{Z}^{\mathrm{der}}(\mathbb{R})_{+} \gamma \alpha \cdot h_{0} \\
& =\Gamma \backslash \Gamma \gamma \gamma^{-1} \boldsymbol{G}_{Z}^{\mathrm{der}}(\mathbb{R})^{+} \gamma \alpha \cdot h_{0} \\
& =\pi_{h_{\alpha}}\left(\Gamma \backslash \Gamma \gamma \boldsymbol{G}_{Z, \gamma}^{\mathrm{der}}(\mathbb{R})^{+}\right)
\end{aligned}
$$

where $\boldsymbol{G}_{Z, \gamma}:=\gamma^{-1} \boldsymbol{G}_{Z} \gamma$. If $\Gamma_{Z} \backslash \mathcal{D}_{Z}^{+} \cap C=\varnothing$, then a fortiori $\Gamma_{Z} \backslash \mathcal{D}_{Z}^{+} \cap \pi_{h_{\alpha}}\left(C^{\prime}\right)=\varnothing$ and hence

$$
\Gamma \backslash \Gamma \gamma \boldsymbol{G}_{Z, \gamma}^{\mathrm{der}}(\mathbb{R})^{+} \cap C^{\prime}=\varnothing .
$$

Since $Z$ is of Shimura type, $G_{Z, \gamma}^{\text {der }}$ is of type $\mathcal{K}$, and hence $G_{Z, \gamma}^{\text {der }}(\mathbb{R})^{+}$is of type $\mathcal{H}$. Then by [CU05-1] Lemma 4.4, there exist a proper $\mathbb{Q}$-parabolic subgroup $\boldsymbol{P}$ of $\boldsymbol{G}$ such that

$$
\boldsymbol{G}_{Z, \gamma}^{\mathrm{der}} \subset \boldsymbol{P} .
$$

But by Proposition 4.4 and [EMS97] Lemma 5.1, this cannot happen if $Z$ is non-factor.

\section{Proof of the main Results}

We now have all the necessary ingredients for the proof of the Theorems 1.11 and 1.7.

Let $\mathbb{V}$ be a $\mathbb{Z}$-VHS on a smooth irreducible complex quasi-projective variety $S$ and $\left(\boldsymbol{G}, \mathcal{D}^{+}\right)$ be the associated connected Hodge datum. By part (3.12) of Theorem 3.12, we may and will assume that $\mathbb{V}$ has no isotrivial factors. 


\subsection{Proof of Theorem 1.11.}

We remark first that we can reduce to the case where $G$ is of adjoint type: this results from Remarks 3.6 and 4.3, and the evident compatibility between the canonical measures associated to non-factor special subvarieties of $\Gamma \backslash \mathcal{D}^{+}$and of $\Gamma^{\mathrm{ad}} \backslash \mathcal{D}^{\text {ad, }}{ }^{+}$. By the structure theorem of period maps (Theorem 3.12) we will assume that $G$ is adjoint of non-compact type. Let us fix a fundamental domain $\mathcal{F}$ of $\mathcal{D}^{+}$for the action of $\Gamma$.

\section{Step 1. Construction of the the limit.}

Let $\left(Z_{n}\right)_{n \in \mathbb{N}}$ be a sequence of non-factor special subvarieties of $S$, which are of Shimura type with dominant period maps. We denote by $\left(\boldsymbol{G}_{n}, \mathcal{D}_{n}^{+}\right)_{n \in \mathbb{N}}$ the corresponding sequence of Hodge subdata of $\left(\boldsymbol{G}, \mathcal{D}^{+}\right)$and $\left(W_{n}\right)_{n \in \mathbb{N}}$ the corresponding sequence of non-factor special subvariety of $\operatorname{Hod}_{\Gamma}^{\circ}(S, \mathbb{V})$.

For each $n \in \mathbb{N}$, by the description in Section 4.2 of special subvarieties for a variation of Hodge structure, we can write $W_{n}$ as

$$
W_{n}=\pi_{h_{n}}\left(\Gamma \backslash \Gamma G_{n}^{\text {der }}(\mathbb{R})^{+}\right)
$$

for any $h_{n} \in \mathcal{D}_{n}^{+} \cap \mathcal{F}$. By Theorem 4.5, there exists a compact subset $C$ of $\mathcal{F}$ such that $C \cap \mathcal{D}_{n}^{+} \neq \varnothing$. We can thus choose $h_{n} \in C \subset \mathcal{F}$. Since by assumption $\left(\boldsymbol{G}_{n}, \mathcal{D}_{n}^{+}\right)$is a connected Shimura datum, the $G_{n}^{\text {der }}$ is of type $\mathcal{K}$ and hence $G_{n}^{\text {der }}(\mathbb{R})^{+}$is a type $\mathcal{H}$ connected closed Lie subgroups of $\boldsymbol{G}(\mathbb{R})^{+}$by Lemma 2.3. Let $\left(\mu_{n}\right)_{n \in \mathbb{N}}$ be the sequence in $\mathcal{P}(\Omega)$ of the canonical Borel probability measures supported on $\Gamma \backslash \Gamma G_{n}^{\text {der }}(\mathbb{R})^{+}$. By Theorem 2.5, there exists a connected Lie subgroup $F$ of $G(\mathbb{R})^{+}$of type $\mathcal{H}$ such that after possibly passing to a subsequence

(a) $\left(\mu_{n}\right)_{n \in \mathbb{N}}$ weakly converges to $\mu_{F}$;

(b) $\operatorname{supp}\left(\mu_{n}\right)=\Gamma \backslash \Gamma G_{n}^{\text {der }}(\mathbb{R})^{+} \subset \Gamma \backslash \Gamma F$, for $n \gg 0$;

(c) the sequence $h_{n}$ converges to $h \in C \subset \mathcal{F}$.

Let $\boldsymbol{H}$ be the smallest $\mathbb{Q}$-algebraic subgroup of $\boldsymbol{G}$ such that $F \subset \boldsymbol{H}(\mathbb{R})$. Then again by Lemma $2.3, \boldsymbol{H}$ is of type $\mathcal{K}$ and $\boldsymbol{H}(\mathbb{R})^{+}=F$. The property $(\mathrm{b})$ implies that $\boldsymbol{G}_{n}^{\mathrm{der}}(\mathbb{R})^{+} \subset$ $H(\mathbb{R})^{+}$, for $n \gg 0$. Hence we deduce that

$$
\boldsymbol{G}_{n}^{\mathrm{der}} \subset \boldsymbol{H}, n \gg 0 .
$$

For $n$ big enough, since $Z_{n}$ is a non-factor special subvariety of $S$, by Proposition 4.4, the centralizer $\mathbf{Z}_{\boldsymbol{G}}\left(\boldsymbol{G}_{n}^{\text {der }}\right)(\mathbb{R})$ is compact. In particular the $\mathbb{Q}$-subgroup $\mathbf{Z}_{\boldsymbol{G}}\left(\boldsymbol{G}_{n}^{\text {der }}\right)$ is $\mathbb{Q}$-anisotropic; that is, it contains no non-trivial $\mathbb{Q}$-split torus. Hence $\boldsymbol{H}$ is reductive by [EMS97] Lemma 5.1. Since $\boldsymbol{H}$ is of type $\mathcal{K}$, it follows that $H$ is semi-simple of non-compact type.

Let $\mathcal{D}_{\infty}^{+} \subset \mathcal{D}^{+}$be the $\boldsymbol{H}(\mathbb{R})^{+}$-conjugacy class of $h, W_{\infty}:=\pi_{h}(\Gamma \backslash \Gamma F)$ and $\mu_{\infty}:=\left(\pi_{h}\right)_{*} \mu_{F}$.

Lemma 5.1. The sequence of measures $\left(\left(\pi_{h_{n}}\right)_{*} \mu_{n}\right)_{n \in \mathbb{N}}$ weakly converges to $\mu_{\infty}$. 
Proof. For any continuous function $f$ on $\Gamma \backslash \mathcal{D}^{+}$with compact support, we have

$$
\begin{aligned}
\pi_{h_{n} *} \mu_{n}(f)-\pi_{h *} \mu_{F}(f) & =\mu_{n}\left(f \pi_{h_{n}}\right)-\mu_{F}\left(f \pi_{h}\right) \\
& =\mu_{n}\left(f \pi_{h_{n}}\right)-\mu_{n}\left(f \pi_{h}\right)+\mu_{n}\left(f \pi_{h}\right)-\mu_{F}\left(f \pi_{h}\right) .
\end{aligned}
$$

By property (a), we have $\mu_{n}\left(f \pi_{h}\right)-\mu_{F}\left(f \pi_{h}\right) \rightarrow 0$, as $n \rightarrow \infty$. Since $h_{n} \rightarrow h$ as $n \rightarrow \infty$ by property (c), the sequence $\left(\pi_{h_{n}}\right)_{n \in \mathbb{N}}$ converges to $\pi_{h}$ and uniformly on all compact subsets. Since $\mu_{n}$ are probability measures, we have $\mu_{n}\left(f \pi_{h_{n}}\right)-\mu_{n}\left(f \pi_{h}\right) \rightarrow 0$ as $n \rightarrow \infty$. Hence we have the convergence.

\section{Step 2. Show that $\mathcal{D}_{\infty}^{+}$is "horizontal".}

For this purpose, we recall a basic result of Griffiths on the analyticity of period images.

Theorem 5.2 (Griffiths [Griff70] Theorems 9.5 and 9.6). Let $\bar{S}$ be a smooth projective compactification of $S$ with $\bar{S} \backslash S$ normal crossing divisor. Let $S^{\prime}$ be the union of $S$ with those points at infinity around which the monodromies are of finite order. Then the period map $\psi$ extends holomorphically to a proper map $\psi^{\prime}: S^{\prime} \rightarrow \operatorname{Hod}_{\Gamma}^{\circ}(S, \mathbb{V})$ and the image $\psi^{\prime}\left(S^{\prime}\right)$ contains $\psi(S)$ as the complement of an analytic subvariety.

Lemma 5.3. $W_{\infty}$ is contained in $\psi^{\prime}\left(S^{\prime}\right)$.

Proof. As the period map for each special subvariety $Z_{n}$ of Shimura type is dominant, $\psi\left(Z_{n}\right)$ will necessary be analytically dense in $W_{n}$. Since $\psi^{\prime}$ is closed, we have $W_{n} \subset \psi^{\prime}\left(S^{\prime}\right)$, for all $n \in \mathbb{N}$.

Suppose that $W_{\infty} \backslash \psi^{\prime}\left(S^{\prime}\right) \neq \varnothing$. Let $x \in W_{\infty} \backslash \psi^{\prime}\left(S^{\prime}\right)$ and let $U_{x}$ be an open neighborhood of $x$ such that $U_{x} \cap \psi^{\prime}\left(S^{\prime}\right)=\emptyset$. By the definition of support of measure, $\mu_{\infty}\left(U_{x}\right)>0$. But $\pi_{h_{n} *} \mu_{n}\left(U_{x}\right)=0$ for any $n \in \mathbb{N}$, which contradicts to the convergence of the measures (Lemma 5.1).

Step 3. Show that $\mathcal{D}_{\infty}^{+}$admits a (unique) complex structure for which the canonical family of Hodge structures (that is, the family associated to the adjoint representation of $H^{\text {ad }}$ on the Lie algebra $\left.\operatorname{Lie}(G)\right)$ varies holomorphically.

Fix any big enough integer $n$ such that $G_{n}^{\text {der }} \subset H$. Let $G_{n}=T_{n} G_{n}^{\text {der }}$ be the almost direct product decomposition of $\boldsymbol{G}_{n}$, where $\boldsymbol{T}_{n}$ is the connected center of $\boldsymbol{G}_{n}$.

Proposition 5.4. $T_{n}$ normalizes $\boldsymbol{H}$.

The proof of Proposition 5.4 follows the same strategy as the proof of [Ull07], Theorem 3.15 in the Shimura variety case. It contains some differences since we are working in the non-classical setting. We shall provide all the details in the next section. 
Let us proceed to finish step 3.

Let $H_{n}$ be the algebraic subgroup of $\boldsymbol{G}$ generated by $\boldsymbol{T}_{n}$ and $\boldsymbol{H}$. Then $H_{n}$ is a reductive $\mathbb{Q}$-group. Let $X_{n}$ be the $\boldsymbol{H}_{n}(\mathbb{R})$-conjugacy class of $h_{n}$ and $X_{n}^{+}$be the connected component of $X_{n}$ containing $h_{n}$. Then we have

$$
h_{n}: \mathbb{S} \rightarrow \boldsymbol{G}_{n, \mathbb{R}} \rightarrow \boldsymbol{H}_{n, \mathbb{R}} \rightarrow \boldsymbol{G}_{\mathbb{R}} .
$$

Let $C=h_{n}(\sqrt{-1})$. It is easy to see that the Killing form is a $C$-polarization for the faithful adjoint representation of $\boldsymbol{H}_{n, \mathbb{R}}^{\text {ad }}$ on the Lie algebra $\operatorname{Lie}(\boldsymbol{G})_{\mathbb{R}}$. So $\left(\boldsymbol{H}_{n}, X_{n}\right)$ is a Hodge subdatum of $(\boldsymbol{G}, \mathcal{D})$. In particular, $X_{n}^{+}$admits a unique complex structure for which the canonical family of Hodge structures varies holomorphically and this complex structure on $X_{n}^{+}$is compatible with complex structure on $\mathcal{D}^{+}$.

By step 2, the holomorphic tangent bundle of $X_{n}^{+}$is contained in the holomorphic horizontal tangent bundle of $\mathcal{D}^{+}$, i.e., the canonical holomorphic family of Hodge structures on $X_{n}^{+}$is a variation of Hodge structure; that is, satisfying Griffiths transversality condition:

$$
F^{-1} \operatorname{Lie}\left(\boldsymbol{H}_{n}\right)_{\mathbb{C}}=\operatorname{Lie}\left(\boldsymbol{H}_{n}\right)_{\mathbb{C}} .
$$

Since the Hodge structure $\operatorname{Lie}\left(\boldsymbol{H}_{n}\right)$ is of weight 0 , it must be of type

$$
\{(-1,1),(0,0),(1,-1)\} \text {. }
$$

Hence the subvarieties $S_{n}=\pi_{h_{n}}(\Gamma \backslash \Gamma \boldsymbol{H}) \cong\left(\Gamma \cap \boldsymbol{H}_{n}(\mathbb{R})_{+}\right) \backslash X_{n}^{+}$are special subvarieties of $\operatorname{Hod}_{\Gamma}(S, \mathbb{V})$ of Shimura type, which are also of non-factor type as each of them contains a non-factor special subvariety $W_{n}$ for $n \gg 0$ respectively.

We thus obtained a sequence of probability measures $\left(\pi_{h_{n}}\right)_{*} \mu_{F}$ with support $S_{n}$, which obviously converges to $\mu_{\infty}=\left(\pi_{h}\right)_{*} \mu_{F}$.

Lemma 5.5. The sequence $\left(S_{n}\right)$ stabilizes as $n$ tends to $\infty$. In particular, we have $W_{\infty}=S_{n}$ for any big enough $n$.

Proof. Note that for $n \gg 0$, we have

$$
S_{n} \cong \Gamma \cap \boldsymbol{H}(\mathbb{R})^{+} \backslash \boldsymbol{H}(\mathbb{R})^{+} / \boldsymbol{H}(\mathbb{R})^{+} \cap M_{n},
$$

where $M_{n}$ is the stabilizer of $h_{n}$ in $G(\mathbb{R})$. Let $K_{n}$ be the unique maximal compact subgroup of $G(\mathbb{R})$ containing $M_{n}$. Since $S_{n}$ are horizontal, we have

$$
H(\mathbb{R})^{+} \cap M_{n}=H(\mathbb{R})^{+} \cap K_{n} .
$$

Since $S_{n}$ are locally symmetric spaces, the $K_{n} \cap H(\mathbb{R})^{+}$are maximal compact subgroups of $\boldsymbol{H}(\mathbb{R})^{+}$. In particular, they all conjugate to each other by elements of $\boldsymbol{H}(\mathbb{R})^{+}$. Fix $n_{0} \gg 0$. For any $n \geq n_{0}$, there exists a $g_{n} \in \boldsymbol{G}(\mathbb{R})$ such that $\pi_{g_{n} h_{n_{0}}}\left(\Gamma \backslash \Gamma \boldsymbol{H}(\mathbb{R})^{+}\right)=\pi_{h_{n}}\left(\Gamma \backslash \Gamma \boldsymbol{H}(\mathbb{R})^{+}\right)$. 
And hence there exists a $v_{n} \in \boldsymbol{H}(\mathbb{R})^{+}$such that

$$
g_{n}\left(K_{n_{0}}\right) g_{n}^{-1} \cap \boldsymbol{H}(\mathbb{R})^{+}=v_{n}^{-1}\left(\boldsymbol{H}(\mathbb{R})^{+} \cap K_{n_{0}}\right) v_{n} .
$$

So $v_{n} g_{n}$ normalizes $K_{n_{0}}$ and hence are in $K_{n_{0}}$. Write $g_{n}=v_{n}^{-1} x$ for some $x \in K_{n_{0}}$, we have

$$
S_{n}=\Gamma \backslash \Gamma \boldsymbol{H}(\mathbb{R})^{+} v_{n}^{-1} x h_{n_{0}}=\Gamma \backslash \Gamma \boldsymbol{H}(\mathbb{R})^{+} x h_{n_{0}}=S_{n_{0}} .
$$

The last equality of (4) is because $\boldsymbol{H}(\mathbb{R})^{+} \cap x M_{n_{0}} x^{-1}$ is a maximal compact subgroup of $\boldsymbol{H}(\mathbb{R})^{+}$and

$$
\boldsymbol{H}(\mathbb{R})^{+} \cap x M_{n_{0}} x^{-1} \subset \boldsymbol{H}(\mathbb{R})^{+} \cap x K_{n_{0}} x^{-1}=\boldsymbol{H}(\mathbb{R})^{+} \cap K_{n_{0}}=\boldsymbol{H}(\mathbb{R})^{+} \cap M_{n_{0}} .
$$

The last statement of Theorem 1.11 is then clear.

5.2. Proof of Theorem 1.7. We argue by contradiction. Suppose that $S$ contains infinitely many distinct non-factor special subvarieties that are of Shimura type with dominant period maps and all are maximal among such kind of special subvarieties. Choose any sequence $\left(Z_{n}\right)_{n \in \mathbb{N}}$ in the set of such kind of special subvarieties. Let $\left(W_{n}\right)_{n \in \mathbb{N}}$ be the corresponding sequence of non-factor special subvariety of $\operatorname{Hod}_{\Gamma}^{\circ}(S, \mathbb{V})$ and let $\mu_{W_{n}}$ be the canonical Borel probability measure on $\operatorname{Hod}_{\Gamma}^{\circ}(S, \mathbb{V})$ with $\operatorname{supp}\left(\mu_{W_{n}}\right)=W_{n}$. By possibly passing to a subsequence, Theorem 1.11 tells us that $\mu_{W_{n}}$ is weakly convergent to $\mu_{\infty}$ and $W_{n} \subset W_{\infty}, n \gg 0$ for a non-factor special subvariety $W_{\infty}$ of $\operatorname{Hod}_{\Gamma}^{\circ}(S, \mathbb{V})$ of Shimura type. Hence $Z_{n}$ is contained in some positive irreducible component of $\psi^{-1}\left(W_{\infty}\right)$ for $k \gg 0$. Since $\psi^{-1}\left(W_{\infty}\right)$ has only finitely many irreducible components by Theorem 3.11 and the irreducible components containing some $Z_{n}$ are non-factor special subvarieties $S$ of Shimura type, by maximality of $Z_{n}$, we deduce that the there are only finitely many possibilities for $Z_{n}$ when $n$ is big enough, which is a contradiction.

If $(S, \mathbb{V})$ is not of Shimura type, the union of all non-factor special subvarieties of Shimura type with dominant period maps is a proper closed subvariety of $S$, which contradicts to the assumption. So $\operatorname{Hod}_{\Gamma}^{\circ}(S, \mathbb{V})$ is a connected Shimura variety.

If the period map $\psi: S \rightarrow \operatorname{Hod}_{\Gamma}^{\circ}(S, \mathbb{V})$ is not dominant, then by [Ull07] Theorem 1.3, the union $U$ of non-factor special subvarities of $\operatorname{Hod}_{\Gamma}^{\circ}(S, \mathbb{V})$ contained in $\overline{\psi(S)}^{\mathrm{Zar}}=\psi^{\prime}\left(S^{\prime}\right)$ is a proper closed subvariety of $\overline{\psi(S)}$ Zar . Hence the preimage $\psi^{-1}(U)$ is a proper closed subvariety of $S$ containing all non-factor special subvarieties of $S$, which again contradicts to the assumption. And we finish the proof of Theorem 1.7.

\section{Proof of the Proposition 5.4}

Fix an arbitrary $n \gg 0$ and write $E:=G_{n}^{\text {der }}, T:=T_{n}$. We assume that $E$ is strictly contained in $H$, otherwise there is nothing to show. 
Lemma 6.1. For any $\mathbb{Q}$-simple factor $\boldsymbol{B}$ of $\boldsymbol{H}$, there exists a noncompact $\mathbb{R}$-simple factor $\boldsymbol{L}_{\mathbb{R}}$ of $\boldsymbol{B}_{\mathbb{R}}$ which is normalized by $\alpha\left(\mathbb{U}^{1}\right)$, where $\alpha \in \mathcal{D}_{n}^{+}$is a Hodge generic point and $\mathbb{U}^{1}$ is the circle subgroup of $\mathbb{S}$. And if $\boldsymbol{B}$ is a $\mathbb{Q}$-simple factor of $\boldsymbol{H}$ such that the projections of $\boldsymbol{E}_{\mathbb{R}}$ to all noncompact $\mathbb{R}$-simple factors of $\boldsymbol{B}_{\mathbb{R}}$ are surjective, then $\alpha\left(\mathbb{U}^{1}\right)$ normalizes $\boldsymbol{B}_{\mathbb{R}}$.

Proof. For the first statement, it suffices to find an element $u \in \mathbb{U}^{1}$ of infinite order such that $\alpha(u)$ normalizes a $\mathbb{R}$-simple factor $\boldsymbol{L}_{\mathbb{R}}$ of $\boldsymbol{B}_{\mathbb{R}}$. Let $u$ be any element of $\mathbb{U}^{1}$ of infinite order and we construct a decreasing sequence $\left(\boldsymbol{B}_{n}\right)_{n \in \mathbb{N}}$ of $\mathbb{R}$-algebraic subgroups of $\boldsymbol{H}$ inductively as follows:

$$
\boldsymbol{B}_{0}=\boldsymbol{H}_{\mathbb{R}}
$$

and for $n \geq 1$

$$
\boldsymbol{B}_{n}=\left(\boldsymbol{B}_{n-1} \cap \alpha(u) \boldsymbol{B}_{n-1} \alpha(u)^{-1}\right)^{\circ} .
$$

Note that $\boldsymbol{E}_{\mathbb{R}} \subset \boldsymbol{B}_{n}$ for any $n \geq 0$. So the sequence $\boldsymbol{B}_{n}$ must be stable by dimension reason. We denote the limit by $\boldsymbol{B}_{\infty}$. By construction, the limit is normalized by $\alpha(u)$ hence also normalized by $\alpha\left(\mathbb{U}^{1}\right)$.

Let $\boldsymbol{B}$ be a $\mathbb{Q}$-simple factor of $\boldsymbol{H}$. Since $\boldsymbol{B}$ is $\mathbb{R}$-isotropic and $\mathbf{Z}_{\boldsymbol{G}}(\boldsymbol{E})(\mathbb{R})$ is compact, the projection of $\boldsymbol{E}$ to $\boldsymbol{B}$ is nontrivial. Let $\boldsymbol{A}$ be a $\mathbb{Q}$-simple factor of $\boldsymbol{E}$ such that the projection of $\boldsymbol{A}$ to $\boldsymbol{B}$ is nontrivial. Let $\boldsymbol{F}_{\mathbb{R}}$ be a noncompact $\mathbb{R}$ - simple factor of $\boldsymbol{A}_{\mathbb{R}}$, then there exists a noncompact $\mathbb{R}$-simple factor $\boldsymbol{L}_{\mathbb{R}}$ of $\boldsymbol{B}_{\mathbb{R}}$ such that the projection of $\boldsymbol{F}_{\mathbb{R}}$ to $\boldsymbol{L}_{\mathbb{R}}$ is nontrivial. Since $\alpha\left(\mathbb{U}^{1}\right)$ normalizes $\boldsymbol{F}_{\mathbb{R}}$, the image of the projection is contained in $\boldsymbol{L}_{\mathbb{R}} \cap \boldsymbol{B}_{\infty}$ which is thus noncompact. By the following Sublemma 6.2, we conclude the first statement.

Sublemma 6.2. $\boldsymbol{L}_{\mathbb{R}} \cap \boldsymbol{B}_{\infty}=\boldsymbol{L}_{\mathbb{R}}$.

Proof. Since $\operatorname{Int}\left(\alpha(\sqrt{-1})\right.$ is a Cartan involution of $\boldsymbol{G}_{\mathbb{R}}$ and fixes $\boldsymbol{E}_{\mathbb{R}}$ and $\boldsymbol{B}_{\infty}$, we have Cartan decompositions:

$$
\begin{aligned}
\boldsymbol{G}(\mathbb{R}) & =P K, \\
\boldsymbol{E}(\mathbb{R}) & =(P \cap \boldsymbol{E}(\mathbb{R}))(K \cap \boldsymbol{E}(\mathbb{R})), \\
\boldsymbol{B}_{\infty}(\mathbb{R}) & =\left(P \cap \boldsymbol{B}_{\infty}(\mathbb{R})\right)\left(K \cap \boldsymbol{B}_{\infty}(\mathbb{R})\right),
\end{aligned}
$$

where $K=Z_{\boldsymbol{G}(\mathbb{R})}(\alpha(\sqrt{-1}))$. Let $M$ be the stablizer of $\alpha$ in $G(\mathbb{R})$, then we have

$$
\mathrm{Z}_{G}(\boldsymbol{E})(\mathbb{R}) \subset M \subset K .
$$

By a result of Mostow [Mos55] on self-adjoint groups, for the inclusion of subgroups

$$
E_{\mathbb{R}} \subset H_{\mathbb{R}} \subset G_{\mathbb{R}}
$$

there exists a $g \in G(\mathbb{R})$ such that we have Cartan decompositions:

$$
\begin{aligned}
& \boldsymbol{E}(\mathbb{R})=\left(g P g^{-1} \cap \boldsymbol{E}(\mathbb{R})\right)\left(g K g^{-1} \cap \boldsymbol{E}(\mathbb{R})\right), \\
& \boldsymbol{H}(\mathbb{R})=\left(g P g^{-1} \cap \boldsymbol{H}(\mathbb{R})\right)\left(g K g^{-1} \cap \boldsymbol{H}(\mathbb{R})\right) .
\end{aligned}
$$


As $\boldsymbol{E}(\mathbb{R})$ admits two Cartan decompositions (6) and (8), they are related by an inner automorphism of $\boldsymbol{E}(\mathbb{R})$, i. e., there exists $t \in \boldsymbol{E}(\mathbb{R})$ such that

$$
\begin{aligned}
& g P g^{-1} \cap \boldsymbol{E}(\mathbb{R})=t(P \cap \boldsymbol{E}(\mathbb{R})) t^{-1}, \\
& g K g^{-1} \cap \boldsymbol{E}(\mathbb{R})=t(K \cap \boldsymbol{E}(\mathbb{R})) t^{-1} .
\end{aligned}
$$

Let $\gamma:=t^{-1} g$, then we have

$$
\begin{aligned}
\gamma P \gamma^{-1} \cap \boldsymbol{E}(\mathbb{R}) & =P \cap \boldsymbol{E}(\mathbb{R}), \\
\gamma K \gamma^{-1} \cap \boldsymbol{E}(\mathbb{R}) & =K \cap \boldsymbol{E}(\mathbb{R}) .
\end{aligned}
$$

Write $\gamma=p k$ with $p \in P$ and $k \in K$. For any $p_{1} \in P \cap \boldsymbol{E}(\mathbb{R})$, there exists a $p_{2} \in P$ such that $p_{2}=\gamma^{-1} p_{1} \gamma$. So $p^{-1} p_{1} p=k p_{1} k^{-1} \in P$, which implies that $p^{2} p_{1}=p_{1} p^{2}$, i.e.

$$
p^{2} \in Z_{\boldsymbol{G}(\mathbb{R})}(P \cap \boldsymbol{E}(\mathbb{R})) .
$$

Similarly, we can show that

$$
p^{2} \in Z_{\boldsymbol{G}(\mathbb{R})}(K \cap \boldsymbol{E}(\mathbb{R})) .
$$

So $p^{2} \in Z_{\boldsymbol{G ( \mathbb { R } )}}(\boldsymbol{E}(\mathbb{R})) \subset K$ which implies $p=1$ and $\gamma \in K$. Hence we have

$$
g K g^{-1} \cap \boldsymbol{H}(\mathbb{R})=t K t^{-1} \cap \boldsymbol{H}(\mathbb{R})=t(K \cap \boldsymbol{H}(\mathbb{R})) t^{-1} .
$$

And thus we have Cartan decompositions of $\boldsymbol{H}(\mathbb{R})$ and $\boldsymbol{L}_{\mathbb{R}}(\mathbb{R})$ :

$$
\begin{aligned}
\boldsymbol{H}(\mathbb{R}) & =(P \cap \boldsymbol{H}(\mathbb{R}))(K \cap \boldsymbol{H}(\mathbb{R})), \\
\boldsymbol{L}_{\mathbb{R}}(\mathbb{R}) & =\left(P \cap \boldsymbol{L}_{\mathbb{R}}(\mathbb{R})\right)\left(K \cap \boldsymbol{L}_{\mathbb{R}}(\mathbb{R})\right) .
\end{aligned}
$$

Since $\mathcal{D}_{\infty}^{+}$is "horizontal" as showed by Step 2 in the proof of Theorem 1.11, we can deduce that $K \cap \boldsymbol{H}(\mathbb{R})=M \cap \boldsymbol{H}(\mathbb{R})$. In particular,

$$
\alpha(u)(K \cap \boldsymbol{H}(\mathbb{R})) \alpha(u)^{-1}=K \cap \boldsymbol{H}(\mathbb{R}),
$$

which implies that

$$
K \cap \boldsymbol{B}_{\infty}(\mathbb{R})=K \cap \boldsymbol{H}(\mathbb{R}) .
$$

Therefore

$$
K \cap \boldsymbol{L}_{\mathbb{R}}(\mathbb{R}) \subset \boldsymbol{B}_{\infty}(\mathbb{R}) \cap \boldsymbol{L}_{\mathbb{R}}(\mathbb{R}) \subset \boldsymbol{L}_{\mathbb{R}}(\mathbb{R}) .
$$

Since $\boldsymbol{L}_{\mathbb{R}}(\mathbb{R})$ is simple and noncompact, the subgroup $K \cap \boldsymbol{L}_{\mathbb{R}}(\mathbb{R})$ is a maximal proper closed subgroup of $\boldsymbol{L}_{\mathbb{R}}(\mathbb{R})$. Hence we have

$$
\boldsymbol{B}_{\infty}(\mathbb{R}) \cap \boldsymbol{L}_{\mathbb{R}}(\mathbb{R})=\boldsymbol{L}_{\mathbb{R}}(\mathbb{R}) .
$$

This finishes the proof of the sublemma.

Now let us prove the second statement of Lemma 6.1. Let $\boldsymbol{B}$ be a $\mathbb{Q}$-simple factor of $\boldsymbol{H}$ such that the projections of $\boldsymbol{E}_{\mathbb{R}}$ to all noncompact $\mathbb{R}$-simple factors of $\boldsymbol{B}_{\mathbb{R}}$ are surjective. If $\boldsymbol{L}_{\mathbb{R}}$ is a compact $\mathbb{R}$-simple factor of $\boldsymbol{B}_{\mathbb{R}}$, then $\boldsymbol{L}_{\mathbb{R}}=K \cap \boldsymbol{L}_{\mathbb{R}}=M \cap \boldsymbol{L}_{\mathbb{R}}$ and hence $\boldsymbol{L}_{\mathbb{R}}$ is normalized 
by $\alpha\left(\mathbb{U}^{1}\right)$. If $\boldsymbol{L}_{\mathbb{R}}$ is a noncompact $\mathbb{R}$-simple factor of $\boldsymbol{B}_{\mathbb{R}}$, by assumption the projection of $\boldsymbol{E}$ to $\boldsymbol{L}_{\mathbb{R}}$ is surjective, from which we deduce that $\boldsymbol{B}_{\infty}(\mathbb{R}) \cap \boldsymbol{L}_{\mathbb{R}}(\mathbb{R})$ is noncompact, and thus equals to $\boldsymbol{L}_{\mathbb{R}}(\mathbb{R})$. So $\boldsymbol{L}_{\mathbb{R}}$ is again normalized by $\alpha\left(\mathbb{U}^{1}\right)$. Therefore $\boldsymbol{B}_{\mathbb{R}}$ is normalized by $\alpha\left(\mathbb{U}^{1}\right)$ and we finish the proof of Lemma 6.1.

Let $\mathcal{S}$ be the poset

$$
\mathcal{S}=\{\boldsymbol{F} \subset \boldsymbol{G} \mid \boldsymbol{F} \text { is a semisimple } \mathbb{Q} \text {-subgroup of type } \mathcal{K} \text { and } \boldsymbol{E} \subsetneq \boldsymbol{F} \subset \boldsymbol{H}\}
$$

with the partial oder given by inclusion.

Lemma 6.3. In order to prove Proposition 5.4, it suffices to assume that $\boldsymbol{H}$ is a minimal element of $\mathcal{S}$.

Proof. Let $\boldsymbol{F}$ be a minimal element of $\mathcal{S}$. By assumption, $\boldsymbol{T}$ normalizes $\boldsymbol{F}$. We have an almost direct product decomposition $\boldsymbol{T}=(\boldsymbol{T} \cap \boldsymbol{F}) \boldsymbol{T}^{\prime}$ with $\boldsymbol{T}^{\prime}$ centralizes $\boldsymbol{F}$. The algebraic subgroup $F^{\prime}$ generated by $F$ and $T$ is reductive and has an almost direct product decomposition $F^{\prime}=$ $T^{\prime} F\left(F=F^{\prime \text { der }}\right)$. Let $\mathcal{D}^{\prime}$ be the $F^{\prime}(\mathbb{R})$-conjugacy class of $\alpha$. Note that $\mathcal{D}^{\prime+}$ is automatically "horizontal" as it is contained in $\mathcal{D}_{\infty}^{+}$. Hence by the same reasoning as in the last part of the proof of Theorem 1.11, $\left(\boldsymbol{F}^{\prime}, \mathcal{D}^{\prime}\right)$ is a Shimura datum. It is easy to see that $\boldsymbol{F}^{\prime}$ is the generic Mumford-Tate group of $\mathcal{D}^{\prime}$. Let $\alpha^{\prime}$ be a Hodge generic point of $\mathcal{D}_{n}^{\prime}$ and replace the Shimura datum $(\boldsymbol{G}, \mathcal{D})$ by $\left(\boldsymbol{F}^{\prime}, \mathcal{D}^{\prime}\right), \boldsymbol{T}$ by $\boldsymbol{T}^{\prime}$ and $\alpha$ by $\alpha^{\prime}$. We thus reduce the proof of Proposition 5.4 to the inclusion $\boldsymbol{F} \subset \boldsymbol{H}$. And after iterating the above procedure finitely many times, the Proposition 5.4 follows.

We suppose now $\boldsymbol{H}$ is minimal in the set $\mathcal{S}$ and proceed to finish the proof of the Proposition 5.4.

Let $\boldsymbol{T}^{\prime}=\boldsymbol{T} \cap \mathrm{N}_{G}(\boldsymbol{H})^{\circ}$ and write $\boldsymbol{T}=\boldsymbol{T}^{\prime} \boldsymbol{T}^{\prime \prime}$ as an almost direct product. Suppose that $\boldsymbol{T}^{\prime \prime}$ is nontrivial. Note that $\alpha\left(\mathbb{U}^{1}\right)=\alpha(\mathbb{S})$ is not contained in $\boldsymbol{T}^{\prime}(\mathbb{R}) \boldsymbol{E}(\mathbb{R})$ as $\boldsymbol{G}$ is adjoint and $\boldsymbol{G}_{n}$ is the generic Mumford-Tate group of $\mathcal{D}_{n}^{+}$. We can choose $b=a g \in \alpha\left(\mathbb{U}^{1}\right)$ with $g \in \boldsymbol{E}(\mathbb{R})$ and $a=a^{\prime} a^{\prime \prime} \in \boldsymbol{T}(\mathbb{R})$ such that $a^{\prime} \in \boldsymbol{T}^{\prime}(\mathbb{R}), a^{\prime \prime} \in \boldsymbol{T}^{\prime \prime}(\mathbb{R})$ and $a^{\prime \prime} \notin \boldsymbol{T}^{\prime}(\mathbb{R}) \cap \boldsymbol{T}^{\prime \prime}(\mathbb{R})$.

Since $T^{\prime}(\mathbb{Q})\left(\operatorname{resp} . T^{\prime \prime}(\mathbb{Q})\right)$ is dense in $T^{\prime}(\mathbb{R})\left(\operatorname{resp} . T^{\prime \prime}(\mathbb{R})\right)$ for the usual topology, we can find a sequence $\left(a_{n}=a_{n}^{\prime} a_{n}^{\prime \prime} \in \boldsymbol{T}(\mathbb{Q})\right)_{n \in \mathbb{N}}$ such that $a_{n}^{\prime} \in \boldsymbol{T}^{\prime}(\mathbb{Q})\left(\right.$ resp. $\left.a_{n}^{\prime \prime} \in \boldsymbol{T}^{\prime \prime}(\mathbb{Q})\right)$ converges to $a^{\prime}$ (resp. $\left.a^{\prime \prime}\right)$. We can also assume that $a_{n}^{\prime \prime} \notin \boldsymbol{T}^{\prime}(\mathbb{R}) \cap \boldsymbol{T}^{\prime \prime}(\mathbb{R})$, for all $n \in \mathbb{N}$.

Now consider $\boldsymbol{H}_{n}^{\prime}:=\left(a_{n} \boldsymbol{H} a_{n}^{-1} \cap \boldsymbol{H}\right)^{\circ}$. As $\boldsymbol{H}_{n}^{\prime}$ contains $\boldsymbol{E}$, it is reductive by [EMS97] Lemma 5.1. We have an almost direct product decomposition

$$
\boldsymbol{H}_{n}^{\prime}=\boldsymbol{H}_{n}^{\mathrm{nc}} \boldsymbol{H}_{n}^{\prime \prime}
$$

where $\boldsymbol{H}_{n}^{\mathrm{nc}}$ is the almost direct product of $\mathbb{Q}$-simple factors of noncompact type of $\boldsymbol{H}_{n}^{\prime}$ and $\boldsymbol{H}_{n}^{\prime \prime}$ is the almost direct product of the remaining factors, so in particular $\boldsymbol{H}_{n}^{\mathrm{nc}} \in \mathcal{S}$. Note that 
the projection of $\boldsymbol{E}$ to $\boldsymbol{H}_{n}^{\prime \prime}$ is trivial, so

$$
\boldsymbol{H}_{n}^{\prime \prime}(\mathbb{R}) \subset \mathbf{Z}_{\boldsymbol{G}}(\boldsymbol{E})(\mathbb{R}) \subset M \cap \boldsymbol{H}(\mathbb{R})=K \cap \boldsymbol{H}(\mathbb{R}) .
$$

Therefore

$$
\boldsymbol{E} \subset \boldsymbol{H}_{n}^{\mathrm{nc}} \subset \boldsymbol{H} .
$$

By minimality of $\boldsymbol{H}$ and $a_{n}^{\prime \prime} \notin \boldsymbol{T}^{\prime}(\mathbb{Q})$, we have $\boldsymbol{H}_{n}^{\mathrm{nc}}=\boldsymbol{E}$ and

$$
\boldsymbol{H}_{n}^{\prime}=\boldsymbol{E} \boldsymbol{H}_{n}^{\prime \prime} \text {. }
$$

Let $n$ tend to infinity, we deduce that every element of $\left(a \boldsymbol{H}(\mathbb{R}) a^{-1} \cap \boldsymbol{H}(\mathbb{R})\right)^{+}$can be written as a product of an elements of $\boldsymbol{E}(\mathbb{R})$ and an element of $M \cap \boldsymbol{H}(\mathbb{R})$.

Let $\boldsymbol{B}$ be a $\mathbb{Q}$-simple factor of $\boldsymbol{H}$, by Lemma 6.1, there exists a noncompact $\mathbb{R}$-simple factor $\boldsymbol{L}_{\mathbb{R}}$ of $\boldsymbol{B}_{\mathbb{R}}$ which is normalized by $\alpha\left(\mathbb{U}^{1}\right)$. Since $b \in \alpha\left(\mathbb{U}^{1}\right)$ and $a \boldsymbol{H}(\mathbb{R}) a^{-1} \cap \boldsymbol{H}(\mathbb{R})=$ $b \boldsymbol{H}(\mathbb{R}) b^{-1} \cap \boldsymbol{H}(\mathbb{R})$, we have

$$
\boldsymbol{L}_{\mathbb{R}}(\mathbb{R})^{+} \subset\left(a \boldsymbol{H}(\mathbb{R}) a^{-1} \cap \boldsymbol{H}(\mathbb{R})\right)^{+},
$$

which implies that $E_{\mathbb{R}}$ projects surjectively onto $\boldsymbol{L}_{\mathbb{R}}$. In particular, $\boldsymbol{L}_{\mathbb{R}}$ is also a $\mathbb{R}$-simple factor of $\boldsymbol{E}_{\mathbb{R}}$.

Sublemma 6.4. The smallest $\mathbb{Q}$-algebraic subgroup $\boldsymbol{F}$ of $\boldsymbol{G}$ which contains $\boldsymbol{L}_{\mathbb{R}}$ is $\boldsymbol{B}$.

Proof. Let $h \in \boldsymbol{B}(\mathbb{Q})$. Then $\boldsymbol{L}_{\mathbb{R}} \subset h \boldsymbol{F}_{\mathbb{R}} h^{-1}$. Since $h \boldsymbol{F} h^{-1}$ is a $\mathbb{Q}$-subgroup of $\boldsymbol{G}$, we have $\boldsymbol{F} \subset h \boldsymbol{F}_{\mathbb{R}} h^{-1}$. So $\boldsymbol{F}$ is a normal subgroup of $\boldsymbol{B}$ and hence equals to $\boldsymbol{B}$ as $\boldsymbol{B}$ is $\mathbb{Q}$-simple.

By Sublemma 6.4, $\boldsymbol{B}$ is contained in $\boldsymbol{E}$. In particular, the projection of $\boldsymbol{E}_{\mathbb{R}}$ to any noncompact $\mathbb{R}$-simple factor of $\boldsymbol{B}_{\mathbb{R}}$ is surjective. By Lemma 6.1, $\alpha\left(\mathbb{U}^{1}\right)$ normalizes $\boldsymbol{B}_{\mathbb{R}}$ and hence normalizes $\boldsymbol{H}_{\mathbb{R}}$, i. e.,

$$
\alpha\left(\mathbb{U}^{1}\right) \subset \mathbf{N}_{G}(H)_{\mathbb{R}}
$$

And thererfore

$$
\boldsymbol{G}_{n} \subset \mathrm{N}_{\boldsymbol{G}}(\boldsymbol{H}) .
$$

In particular, $\boldsymbol{T}_{n} \subset \mathrm{N}_{G}(H)$ which contradicts to our assumption that $T_{n}^{\prime \prime}$ is nontrivial. This completes the proof of Proposition 5.4.

\section{REFERENCES}

[An92] Y. André: Mumford-Tate groups of mixed Hodge structures and the theorem of the fixed part, Compositio Math. 82 (1992), no. 1, 1-24. 12, 14

[AGHM18] F. Andreatta; E. Goren; B. Howard; K. Madapusi Pera: Faltings heights of abelian varieties with complex multiplication, Ann. of Math. (2) 187 (2018), no. 2, 391-531. 1

[BKT18] B. Bakker; B. Klingler; J. Tsimerman: Tame topology of arithmetic quotients and algebraicity of Hodge locus, arXiv:1810.04801. 6, 8, 10, 12

[CDK95] E. Cattani, P. Deligne, A. Kaplan: On the locus of Hodge classes, J. of AMS, 8 (1995), 483-506. 2 
[CMP17] J. Carlson; S. Müller-Stach; C. Peters: Period mappings and period domains, Second edition. Cambridge Studies in Advanced Mathematics, 168. Cambridge University Press, Cambridge, 2017. xiv+562 pp. 8, 9, $11,12,13$

[CU05-1] L. Clozel, E. Ullmo: Équidistribution de sous-variétés spéciales, Ann. of Math. (2) 161 (2005), no. 3, 1571-1588. 1, 5, 6, 7, 8, 15, 16

[CU05-2] L. Clozel, E. Ullmo: Équidistribution de mesures algébriques, Compos. Math. 141 (2005), no. 5, 1255-1309. 7

[DM91] S. G. Dani, G. A. Margulis: Asymptotic behaviour of trajectories of unipotent flows on homogeneous spaces, Proc. Indian Acad. Sci. Math. Sci. 101 (1991), no. 1, 1-17. 6, 15, 16

[EMS97] A. Eskin; S. Mozes; N. Shah: Non-divergence of translates of certain algebraic measures, Geom. Funct. Anal. 7 (1997), no. 1, 48-80. 16, 17, 23

[GGK12] M. Green, P. Griffiths, M. Kerr: Mumford-Tate groups and domains. Their geometry and arithmetic, Annals of Mathematics Studies 183, Princeton University Press, 2012. 3, 8

[Griff70] P. Griffiths: Periods of integrals on algebraic manifolds. III. Some global differential-geometric properties of the period mapping, Inst. Hautes Études Sci. Publ. Math. No. 38 (1970), 125-180. 18

[GRT14] P. Griffiths; C. Robles; D. Toledo: Quotients of non-classical flag domains are not algebraic, Algebr. Geom. 1 (2014), no. 1, 1-13. 9

[Gao17] Z. Gao: Towards the Andre-Oort conjecture for mixed Shimura varieties: the Ax-Lindemann theorem and lower bounds for Galois orbits of special points, J. Reine Angew. Math. 732 (2017), 85-146. 1

[K17] B. Klingler, Hodge locus and atypical intersections: conjectures, to appear in Motives and complex multiplication. 1, 4, 5, 8

[KO19] B. Klingler, A. Otwinowska: On the closure of the positive Hodge locus, arXiv:1901.10003v2. 5

[KUY16] B. Klingler, E. Ullmo, A. Yafaev: The hyperbolic Ax-Lindemann-Weierstrass conjecture, Publ. Math. Inst. Hautes Études Sci. 123 (2016), 333-360. 1

[KUY18] B. Klingler, E. Ullmo, A. Yafaev: Bi-algebraic geometry and the André-Oort conjecture, Algebraic geometry: Salt Lake City 2015, 319-359, Proc. Sympos. Pure Math., 97.2, Amer. Math. Soc., Providence, RI, 2018. 4

[Mil05] J. Milne: Introduction to Shimura varieties. Harmonic analysis, the trace formula, and Shimura varieties, 265-378, Clay Math. Proc., 4, Amer. Math. Soc., Providence, RI, 2005. 3, 4, 9

[Mil17] J. Milne: Algebraic groups. The theory of group schemes of finite type over a field, Cambridge Studies in Advanced Mathematics, 170. Cambridge University Press, Cambridge, 2017. xvi+644 pp. 7

[Mos55] G. D. Mostow: Self-adjoint groups, Ann. of Math. (2) 62 (1955), 44-55. 21

[MS95] S. Mozes, N. Shah: On the space of ergodic invariant measures of unipotent flows, Ergodic Theory Dynam. Systems 15 (1995), no. 1, 149-159. 6, 7, 8

[PT14] J. Pila; J. Tsimerman: Ax-Lindemann for $\mathcal{A}_{g}$, Ann. of Math. (2) 179 (2014), no. 2, 659-681. 1

[Rat91-1] M. Ratner: On Raghunathan's measure conjecture, Ann. of Math. (2) 134 (1991), no. 3, 545-607. 6, 7

[Rat91-2] M. Ratner: Raghunathan's topological conjecture and distributions of unipotent flows, Duke Math. J. 63 (1991), no. 1, 235-280. 6, 7

[Ray88] M. Raynaud: Sous-variétés d'une variété abélienne et points de torsion, in Arithmetic and Geometry, Vol. I, Progress in Math. 35 (1988). 1

[Sch73] W. Schmid: Variation of Hodge structure: the singularities of the period mapping, Invent. Math. 22 (1973), 211-319. 2

[Tsi18] J. Tsimerman: The André-Oort conjecture for $\mathcal{A}_{g}$, Ann. of Math. (2) 187 (2018), no. 2, 379-390. 1

[Ull07] E. Ullmo: Équidistribution de sous-variétśs spéciales II, J. Reine Angew. Math. 606 (2007), 193-216. 1, 5, $6,7,13,15,18,20$ 
[vdD98] L. van den Dries: Tame topology and o-minimal structures, London Mathematical Society Lecture Note Series, 248. Cambridge University Press, Cambridge, 1998. x+180 pp. 12, 13

[YZ18] X. Yuan; S. Zhang: On the averaged Colmez conjecture, Ann. of Math. (2) 187 (2018), no. 2, 533-638. 1

Université de Paris (Institut de mathématiques de Jussieu - Paris Rive Gauche, Paris)

Email address: jiaming.chen@imj-prg.fr 